\title{
Modelling Revenue Generation in a Dynamically Priced Mobile Telephony Service
}

HAN W ANG, National University of Ireland Galway, Ireland

DAMIEN FAY, Data Science Institute, Bournemouth, University, UK

KEN BROWN, University College Cork, Ireland

LIAM KILMARTIN, National University of Ireland Galway, Ireland

\begin{abstract}
Dynamic pricing has been used extensively in specific markets for many years but recent years has seen an interest in the utilization of this approach for the deployment of novel and attractive tariff structures for mobile communication services. This paper describes the development and operation of an Agent Based Model (ABM) for subscriber behavior in a dynamically priced mobile telephony network. The design of the $\mathrm{ABM}$ was based on an analysis of real Call Detail Records (CDRs) recorded in a Uganda mobile telephony network in which dynamic pricing was deployed. The ABM includes components which simulate subscriber calling behavior, mobility within the network and social linkages. Using this model, this paper reports on an investigation of a number of alternative strategies for the dynamic pricing algorithm which indicate that the network operator will likely experience revenue losses ranging from a $5 \%$, when the pricing algorithm is based on offering high value subscriber cohort enhanced random discounts compared to a lower value subscriber cohort, to $30 \%$, when the priding algorithm results in the discount on offer in a cell being inversely proportional to the contemporary cell load. Additionally, the model appears to suggest that the use of optimization algorithms to control the level of discount offered in cells would likely result in discount simply converging to a "no-discount" scenario. Finally, commentary is offered on additional factors which need to be considered when interpreting the results of this work such as the impact of subscriber churn on the size of the subscriber base and the technical and marketing challenges of deploying the various dynamic pricing algorithms which have been investigated.
\end{abstract}

General Terms: Economics, Design, Algorithms, Performance

Additional Key Words and Phrases: Agent-based model, revenue optimization, dynamic pricing, mobile network services

\section{INTRODUCTION}

Mobile phone penetration levels have experienced exponential growth over the last decade, growing from $34 \%$ in 2005 to over $96 \%$ in February 2013 according to ITU-T statistics [ITU. 2014]. This growth has been particularly noteworthy in regions which are categorized as "developing" by the ITU-T, displaying a growth from 1.2 billion subscriptions ( $23 \%$ penetration level) to over 5.2 billion subscriptions (89\% penetration) in the same time period. As a result, there has been an increased commercial focus for mobile phone networking companies on such developing markets given its potential for revenue generation into the future. Africa in particular is a region which clearly illustrates these characteristics with the ITU-T estimating that mobile phone subscriptions having grown from approximately 90 million ( $12 \%$ penetration rate) in 2005 to nearly 550 million (63\% penetration rate) in 2013 and with an estimate that the subscriber base in Africa will reach over 1 billion subscribers by 2016 [Donovan and Martin. 2014]. One key characteristic which particularly distinguishes mobile network subscribers in the African market (and indeed in developing markets in general) is a very high degree of price sensitivity amongst subscribers. For example, it is not uncommon in such countries that the vast majority of the subscriber base sign up for pre-paid services. As an example, in

This research is funded under the Enterprise Partnership Program of the Irish Research Council (IRC) with co-funding from Tango Telecom Limited.

Author's addresses: H. Wang and L. Kilmartin, Electrical \& Electronic Engineering, College of Engineering and Informatics, National University of Ireland Galway, Ireland; D. Fay, Data Science Institute, Bournemouth University, Poole House, P 317 Talbot Campus, Fern Barrow Poole, Dorset, BH12 5BB, UK; K. Brown, Department of Computer Science, University College Cork, College Road, Cork, Ireland. 
Uganda, it is estimated that over 99\% of mobile phone subscriptions are for pre-paid services [UCC. 2012]. Given this extremely competitive environment, mobile network service providers operating in many developing countries have been forced to developed and deploy a wide variety of strategies and services in order to retain subscribers and attempt to encourage increases in use of network services [SAS. 2010].

One such strategy which has seen significant growth in terms of deployments in African markets in particular in recent years is the use of real time dynamic pricing for voice, and to a much smaller degree, non-voices services. A Dynamic Pricing Service (DPS) [Fitkov-Norris and Khanifar. 2000; Fitkov-Norris and Khanifar. 2001; Olivré. 2004] is a tariffing tool which results in the cost associated with a voice call being varied by the network in real-time based on a combination of subscriber location and time of day. In a network implementing a DPS, the most common deployment model is one where subscribers in the network are provided with a real time indication of a discounted tariff (most typically in the form of a discount on some nominal tariff level) being offered for certain categories of voice calls (e.g. "on-net", "off-net" or perhaps "national" calls). This discount not only varies throughout the day but will also typically vary depending on the particular mobile network cell which is currently serving the subscriber. The manner in which the dynamic discounting factor is controlled is key to ensuring that the deployment of a DPS is successful from a network operator's perspective. However, it is first important to understand that there may be multiple (sometimes competing) drivers motivating a network operator to deploy a DPS in their network.

The most obvious motivator for a network operator to deploy a DPS is to motivate subscribers to increase their usage of the network and hence ideally to achieve a maximization of revenue generated from the resultant voice calls across the base of subscribers who opt-in to utilize the service. However, this is a quite a complex challenge to achieve given the huge diversities in subscriber behavior which will be observed in any reasonably sized network. A poorly operating algorithm which is used to control the discounting factors within a DPS may result in significant increases in call attempts but may also result in a reduction in the overall revenue generated for the network operator. Despite claims made in marketing material of companies offering DPS capabilities to network operators, very little research has been reported in the literature on the ability of DPS systems to increase and, ideally maximize, overall network operator revenue. A second motivator for the use of a DPS in a mobile network is to address the issue of subscriber churn. With careful marketing, the launch of a DPS in a mobile network can be a powerful tool in (i) anchoring a significant percentage of its subscriber base through opting in to the new service and (ii) attracting large number of new subscribers into using the DPS from competing networks. It should however be noted at this point that this justification for the deployment of a DPS is not necessarily complimentary to aim of maximizing revenue generation. It is quite conceivable that the typical levels of discount which may have to be offered from a purely marketing perspective (in order to make usage of the DPS attractive to subscribers) may not necessarily result in revenue optimization. A final motivation for the deployment of a DPS in a network is as a tool to manage traffic load in cells. In such a case, the discount on offer would be inversely related to the utilization or traffic load in a cell (i.e. Thus when cell load is low the offered discount would be higher thus incentivizing subscribers to make voice calls. When cell load is high the opposite would be the case). Once again however considerations of other effects need to be taken into account when using a DPS in 
Investigating the Revenue Generation Impact of Dynamic Pricing Algorithms for Mobile Voice Services $1: 3$

this way. For example, there may be certain times of the day (e.g. middle of the night) or certain cell locations (e.g. remote cell locations) where such a strategy will simply not have any effect, other than to reduce revenue generation for the operator. Another impact which would have to be considered is how such a strategy might impact on the available voice bandwidth in a cell site to other subscribers of the network who have not opted into the DPS (e.g. post-paid subscribers). In particular, post-paid subscribers in generally deliver much higher Average-Revenue-Per-User (ARPU) compared to pre-paid subscriber. Hence an operator would not wish for such subscribers to be blocked from making calls due to all available cell bandwidth being used to service lower revenue generating pre-paid subscribers who have opted into the DPS.

The focus of this paper is the development of a realistic model of subscriber behavior in a dynamic pricing environment for voice calls. This Agent Based Model (ABM) has its foundation in a Call Detail Record (CDR) dataset gathered from a real deployment of a DPS in the African country of Uganda in 2010. Traditional statistical models which might be applied to this problem have limitations in terms of the identification individual subscribers' calling patterns and the evolution of subscribers' social and mobility patterns [Gonzalez et al. 2008; Hidalgo and Rodriguez-Sickert. 2008; Isaacman et al. 2012]. Whilst some previous work, such as [Fitkov-Norris and Khanifar. 2000; Fitkov-Norris and Khanifar. 2001; Olivré. 2004], did examine the issue of the impact of dynamic pricing in voice networks, to our knowledge this is the first paper to examine the issue of modeling subscriber behavior by utilizing a data driven approach (i.e. CDRs) from a real network implementing a DPS rather than from a theoretical simulation approach. In addition to the development of the agent based model, the paper also describes investigations which we have carried out using a variety of algorithms to control the discounting element of the service. The purpose of these investigations is to determine how the revenue generation capabilities of the various algorithms compare when applied to a simulated population of subscribers. The results of these investigations provide insights into the ability of dynamic pricing services to actually deliver on their aims to network operators who deploy them.

The remainder of this paper is organized as follows. Section 2 discusses the related work in the research literature covering dynamic pricing, CDR data set mining and analysis and agent based modelling. Section 3 describes the CDR data set which was the basis of this work and it provides an overview of the initial analysis which was completed on this data. It also introduces the structure and operation of our agent based model and in particular the use of the CDR data set in its design. Section 4 is focused on the revenue generation performance of the various discounting algorithms which were investigated using the developed model. In the final section of the paper, we present the conclusions of our study and outline our work which is being carried out in this area using the developed model.

\section{RELATED WORK}

This section of the paper provides a review of related work in a number of key areas relating to this paper. We first provide an overview on research which has been completed on the area of dynamic pricing in different industries and, in particular, a limited number of papers which have investigate its use for tariffing in mobile network services. Secondly, we present a review of some key papers relating to the 
analysis of large data sets formed from CDRs from mobile networks. Recent years have seen significant interest in this field for many reasons and in particular we review previous worked reported relating to the development of models based behavior observed in such data sets. A final topic which is of significant importance to this paper is the area of ABMs and the final part of this literature review section provides an overview of this general area and how ABMs have been applied in a variety of application spaces.

\subsection{Dynamic Pricing}

Dynamic Pricing is used to adjust the price of a service or product in order to change the demand response from users and, as a result, to increase the profit gained from the service/product [Christ. 2011; Dolgui and Proth. 2010]. This form of revenue management strategy has been used in many different industries. There exists a significant body of research, commonly theoretical in nature, on the topic of dynamic pricing particularly in the field of operations research [Levin et al. 2007; Nasiry and Popescu. 2011; Popescu and Wu. 2007].

There have also been numerous studies of a more applied nature on the application of dynamic pricing in a variety of scenarios. Probably the most commonly encountered example of the use of dynamic pricing is in the airline industry where passengers can usually get cheaper flight tickets in the off-season, or if booked well in advance of the flight date, as examined in [McAfee and Velde. 2006]. Similarly, the hotel industry also dynamically changes room prices based on occupancy rates of a hotel, a topic which was investigated in [Bayoumi et al. 2013]. In the food retail industry, the pricing of perishable foods is also suitable for the application of dynamic pricing, since the consumer's purchasing demand may change based on the "quality" of the product (i.e. which often will be directly related to its remaining shelf-life). Chung and Li investigated the impact of frequency of discount during a product's selling period on retailer performance [Chung and Li. 2013]. In the area of electricity tariffing, the introduction of smart meters [Faruqui. 2010; Gerwen et al. 2006; Molina-Markham et al. 2012] which are capable of accurately measuring when and how much energy is used by a consumer in real time [Faruqui and George. 2005] has created opportunities for introduction of dynamic pricing. [E3. 2006; Jessoe et al. 2012] examine the potential for electricity suppliers to offer customers dynamic tariffs depending on the time of day at which electricity is consumed (an approach which is commonly termed Time of Use pricing (TOU)). Whilst TOU tariffing is typically applied to scenarios where the price is set for longer periods of time (i.e. 24 hours or more), another similar dynamic pricing strategy used in the electricity supply industry is Real Time Pricing (RTP) which results in the tariff being varied over much shorter time periods in order for it to reflect the wholesale price of electricity directly, as examined in [Allcott. 2009; Allcott. 2011; Faria and Vale. 2011; Samadi et al. 2010]. Another form of dynamic pricing in the electricity supply industry as examined in [Qin et al. 2009] is Critical Peak Pricing (CPP). This is a hybrid pricing strategy based on TOU and RTP with a pre-set high price during periods of sustained high demand for the product.

Of more specific interest to our work, there is an existing body of work on the application of dynamic pricing for communication network services. A significant proportion of this work has focused on the application of dynamic pricing for internet and data services, such as in [Dimicco et al. 2003; Kannan and Kopalle. 2001; Leloup and Deveaux. 2001; Yaipairoj and Harmantzis. 2004]. Some previous work also has examined the potential application of dynamic pricing specifically for voice services. 
Investigating the Revenue Generation Impact of Dynamic Pricing Algorithms for Mobile Voice Services $1: 5$

Fitkov-Norris and Khanifar [Fitkov-Norris and Khanifar. 2001] proposed one of the first dynamic pricing algorithms for use in mobile voice networks. Although their proposed algorithm was relatively simple, its foundation is well based and centered on a number of widely accepted assumptions. They proposed that a higher price would cause users to shorten their call duration or reduce the number of phone calls attempted. Alternatively, a lower price would encourage the users to make more phone calls or extend their call durations. Fishburn and Odlyzko [Fishburn and Odlyzko. 1998] examine the problem from the perspective of maximizing the revenue generated for network operators. They proposed an approach where the subscriber would be charged a fixed price when the current incoming call rate was greater than an optimal incoming call rate. Mirsarraf and Mansoori [Mirsarraf and Mansoori. 2008] examined the approach from the slightly different perspective of considering the tariffing offered by competitor networks. They proposed a model where this effect was integrated by updating the offered tariff using an algorithm termed "Learning From Competitor's" (LFC) price.

\subsection{Analysis of CDR Datasets}

Recent years, particularly with the general growing interest in the research community on the topic of big data, has seen significant research interest on the analysis of the huge CDR based data sets which are being continuously generated by entities within mobile and fixed line communication networks. The research problems being investigated through the analysis of these data sets is quite diverse.

Many papers have focused on the development of statistical models for a variety of underlying subscriber characteristics such as call frequency and duration [Dasgupta et al. 2008; Willkomm et al. 2008], inter-event time [Candia et al. 2007], call arrival rate [Willkomm, Machiraju, Bolot and Wolisz. 2008] and user mobility [Chaogui et al. 2010; Yuan et al. 2011; Zang and Bolot. 2007] (e.g. cell visit frequency). The application of graph theory based analysis techniques from the complex system domain have also been used in the analysis of such large CDR data sets [Hossmann et al. 2011; Nanavati et al. 2008; Song et al. 2010; Wang et al. 2009; Ye et al. 2009]. When analyzing CDR data sets, the network nodes can be either a subscriber or a cell site and the node may also have a defined position in a Euclidian space. In such situations, geographic space is important as the network topology alone does not contain all the system information. [Barrat et al. 2005; Hayashi. 2006; Kitchin and Dodge. 2000; Liben-Nowell et al. 2005] utilized such an approach and highlighted the important consequence of spatial networks in there being a cost associated with the length of edge. This spatial property is very useful for regional analysis because the distance between two subscribers or two cell sites reflects the connectivity level between these two entities. For example, the gravity model has been widely used to model flows such as the road and airline networks between cities [Barrat et al. 2004; Jung et al. 2008].

Studies have focused on the identification of, or comparisons between, communities or cliques from mobile phone network data sets [Fortunato. 2010; Onnela et al. 2007; Tomar et al. 2010]. A common theme in these studies is that they utilized location information such as the location of cell towers with a significant focus on spatial network analysis. Expert et al. highlighted the issue that most of the studies utilized standard metrics extracted from networks of subscribers which had lost any spatial properties. As a result, the authors focus on the problem of community detection and 
proposed a modularity function adapted to spatial networks. They illustrated that the inclusion of spatial information could reveal hidden structural similarities between nodes [Expert et al. 2011]. Eagle et al. provided a comparison of the behavior of different communities i.e. between rural and urban societies. They demonstrated that individuals change their patterns of communication to increase similarity with their new social environment [Eagle et al. 2009]. In order to detect communities in large networks, Blondel et al. propose a heuristic method to extract community structure based on modularity optimization [Blondel et al. 2008]. They applied this algorithm to identify language based communities in a CDR data set from a Belgian mobile phone network [Walsh and Pozdnoukhov. 2011]. This community detection method is simple and fast compared with Newman's method [Newman. 2006]. Walsh and Pozdnoukhov also applied Blondel's method to explore the temporal evolution and the spatial organization of urban communities [Walsh and Pozdnoukhov. 2011].

In addition to community detection, the detection of important locations is another popular focus for work involving the analysis of CDR data sets (particularly for urban planning and emergent event detection). Isaacman et al. used clustering and regression techniques on a mobile phone data set in order to identify important locations such as subscribers' home and work locations [Isaacman et al. 2011]. Vieira et al. adopted a more general point of view to characterize dense urban areas in order to study social dynamics [Vieira et al. 2010]. Becker et al. presented several ways in which CDRs can be used to provide important information about city dynamics to urban planners, such as the ability to automatically identify residential areas [Becker et al. 2011]. Calabrese et al. analyzed 1 million cell-phone traces and associated their

destinations with social events [Calabrese et al. 2010]. They found that the behavior of people attending an event were strongly correlated to the type of event and that people who live close to an event are preferentially attracted by it. This information is very useful for city management functions such as events management and congestion mitigation. From another perspective, Soto and Frías-Martínez used the Fuzzy c-means clustering algorithm to identify land use in urban areas [Soto and FríasMartínez. 2011].

Apart from spatial network analysis, there are other areas of research based on CDRs such as finding usage groups [Becker et al. 2011], understanding traffic dynamics in cellular data networks [Paul et al. 2011], analyzing urban human mobility [Noulas et al. 2012] and identifying information diffusion in mobile networks [Cebrián et al. 2010].

\subsection{Agent-based Modelling}

As noted previously, one of the primary aims of this work is the development of an Agent based Model for subscriber behavior in a dynamically priced mobile voice network. Hence it is informative to briefly examine the general topic of Agent based Models (ABM) and their application in the existing literature. An ABM is an individual-level modeling system which describes and simulates a system which is designed to model the behavior and interaction of large groups of real-world entities. In recent years, the increase in computing power and storage capacity has facilitated a growth in interest in the research community on the use of ABMs [Axelrod. 1997; Bankes. 2002; Castle and Crooks. 2006; Gilbert. 2008; Nikolai and Madey. 2009]. Compared to model traditionally modelling approaches, an agent-based approach offers more flexibility and can be used to model and simulate discontinuous and nonlinear situations [Parker et al. 2002]. Traditionally, ABM was commonly used in Geographic Information Systems (GIS) to build complex geospatial models [Crooks et 
Investigating the Revenue Generation Impact of Dynamic Pricing Algorithms for Mobile Voice Services $1: 7$

al. 2008]. It has also been used to develop workflow management system (WfMS) which have been used to simulate emergent evacuation flows, traffic flows and customer flows [Bonabeau. 2002; Ehrler et al. 2005; Guo et al. 2008; Tepfenhart et al. 2009]. ABMs have also been used for urban planning [Martínez and Morales. 2012] such as modeling the land-use and land-cover change [Matthews et al. 2007; Parker et al. 2001].

Another application area where ABMs have been applied is in the area of modelling consumer choice and decision making. In [Zhang and Zhang. 2007], Zhang and Zhang proposed the use of an agent to simulate the consumer's purchase decision-making. Lamjed Ben Said et al. used an agent-based approach to create a virtual consumer behavioral model in order to simulate the effects of marketing strategies in a competing market context [Said et al. 2002]. Chappin and Afman et al. developed an agent-based model to simulate lamp purchasing behavior amongst Dutch consumers [Chappin and Afman. 2013]. In [North et al. 2010], North et al. applied agent-based modeling to develop a multi-scale consumer market model.

Agent based modelling has also been used to study various business and financial market phenomena. A comprehensive review of agent-based model applied in finical markets was carried out by Samanidou et al. in [Samanidou et al. 2007]. Rand and Rust [Rand and Rust. 2011] emphasized the advantage of using agent-based approach to simulate complex marketing phenomena from simple decision rules. They also proposed some guidelines and highlighted some examples of how to use agent-based approaches. A more specific example was examine in [Bonabeau. 2002] on how an agent-based approach could be adopted for modelling a dynamic stock market. Agent-based models have also been used in social network analysis [Bergenti et al. 2011; Hamill and Gilbert. 2010; Hamill and Gilbert. 2010; Madey et al. 2003]. Singer et al. proposed to use of ABMs to examine friendship structures in social networks [Singer et al. 2009]. In social epidemiology research, agent-based approaches have also been used to understand causal inference and simulate the exploration of etiologic pathways [El-Sayed et al. 2012]. In social diffusion network

analysis, Schwarz and Ernst proposed an agent-based model for the diffusion of water-saving innovations where the agents are households with certain lifestyles [Schwarz and Ernst. 2009].

However, of direct interest to this paper, only a small number of papers have focused on the application of ABMs to the problem of modelling subscriber behavior in mobile networks. Mohammed proposed the use of an ABM approach to investigate customer retention in the UK mobile market [Hassouna. 2012]. Frías-Martínez et al. used a CDR data set to develop an ABM model to simulate epidemic spread [Frías-Martínez et al. 2011]. Twomey and Cadman introduced the concept of agent-based modeling and presented a business application in a telecoms and media market [Twomey and Cadman. 2002].

\section{AGENT BASED MODEL DESIGN}

In this section, we provide an overview of the process through which the Agent Based Model (ABM) developed in this research was designed. Prior to the initiation of the design phase of the model, the CDR data captured from a DPS deployment in a mobile network in Uganda was analyzed in order to gain some insights into general 
subscriber behavior and in order to develop models for this behavior. We initially provide an overview of the data in this CDR dataset and some important results from this investigation in section 3.1. Section 3.2 then provides a detailed description of the various components sub-models within the ABM and how these were designed based on observations from the CDR dataset analysis.

\subsection{CDR Dataset and Initial Analysis}

The raw data (CDRs) analyzed in this research was captured from a DPS platform in a mobile network in the African country of Uganda for 19 weeks. The records for all prepaid subscriber (who had opted into the DPS) call attempts on each Wednesday were recorded starting from April 28th, 2010 and ending on September 22nd, 2010. Typically the daily CDRs generated by the system represented approximately 6.5 million call attempts involving 2 million unique participants. Fig. 1 provides an overview of subscriber usage of this service from its launch date in late February 2014. These plots illustrate the growth period in subscriber usage of the service after its launch, including the period during which the CDRs which were analyzed in this research were captured (i.e. shaded region of graphs in Fig. 1).
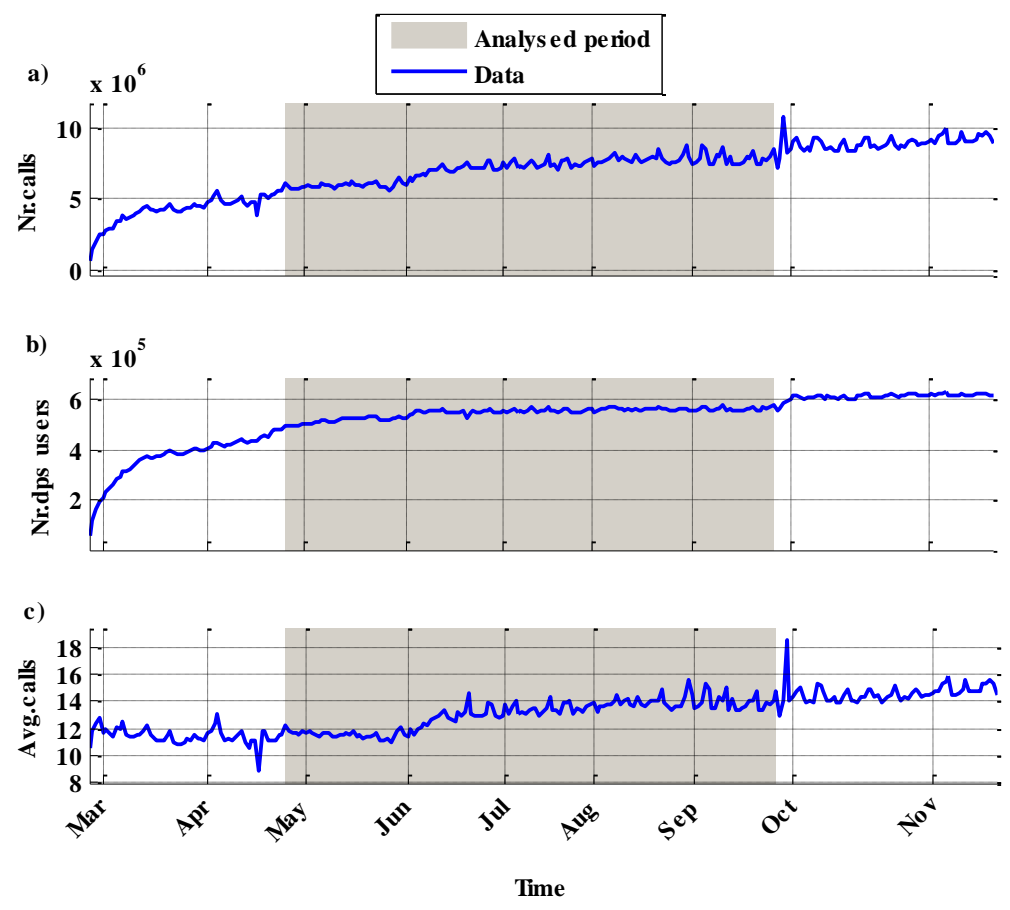

Fig. 1. (a) Number of call attempts through the DPS, (b) Number of subscribers opted into the DPS and (c) Average number of calls per subscriber all displayed for a 9 month time period post service launch.

It was decided to limit the initial analysis of the CDR dataset to the subset of call attempts between prepaid subscribers of the same mobile network (i.e. on-net calls) in order to reduce the number of CDRs to be processed, without any loss of generality. This data set typically consisted of CDRs representing an average of 3.5 million call attempts between approximately 800,000 unique participants each day. An example of the format of the anonymized CDRs from the data set is shown in Table I. The original CDR dataset did not contain information relating to the geographic location of cell sites, however a semi-automated algorithm was used [Wang and Kilmartin. 2014 to provide estimates of the longitude and latitude of each cell site. 
Investigating the Revenue Generation Impact of Dynamic Pricing Algorithms for Mobile Voice Services $1: 9$

\begin{tabular}{|c|c|c|c|c|c|}
\hline & & & & & \\
\hline Stamp & Party ID & Party ID & Tower ID & $\begin{array}{l}\text { DPS } \\
\text { Discount } \\
(\%)\end{array}$ & $\begin{array}{l}\text { Utilization } \\
\text { Factor }\end{array}$ \\
\hline 734628 & 1 & 2 & 265 & 70 & 0.2365 \\
\hline
\end{tabular}

[Wang and Kilmartin. 2014] outlines some of the analysis and associated observations gained in an initial analysis of the CDR data set. One distinct characteristic which was highlighted in this analysis was a high degree of regional heterogeneity in subscriber behavior, mobility and social linkages. In this work, four different geographical regions were defined within Uganda, namely covering the Northern administrative region, the Western and Central administrative regions, the Eastern administrative region and a small region around the capital, Kampala (see Fig. 2 (a) and (b)).

a)

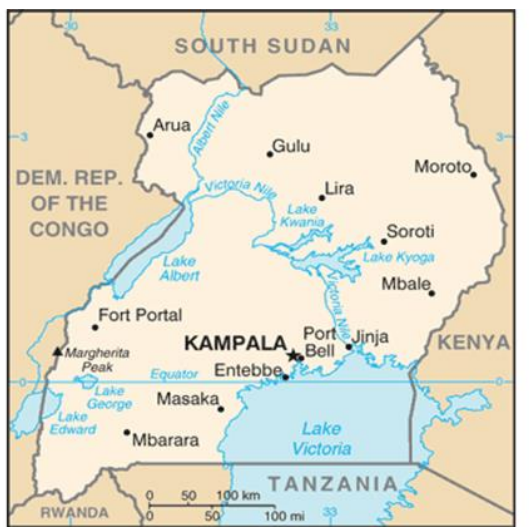

b)

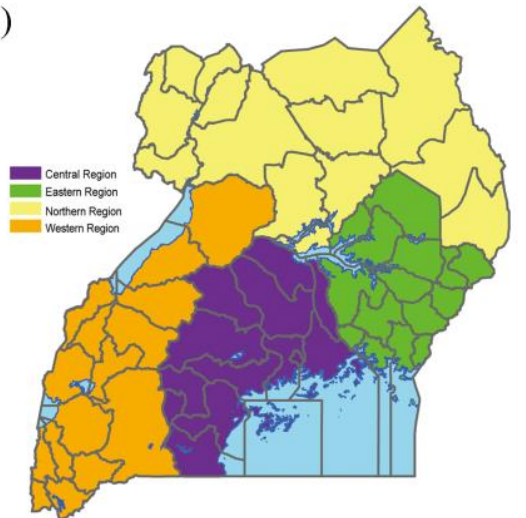

Fig. 2. (a) Geography of Uganda and (b) Administrative regions of Uganda Sources: (a) CIA World Factbook, (b) www.wikipedia.org

This work utilized a graph theory based approach, Mobile Travel Graphs (MTG) to examine subscriber mobility patterns. These clearly illustrated a pattern where the majority of subscribers tended to only travel over very short distances and to remain within their own regions. The work also identified significant similarities in subscribers' calling behavior particularly between regions with similar levels of economic development (i.e. rural versus urban development). Fig. 4 utilizes Mobile Call Graphs [Qi et al. 2008] (MCG) for each of these four regions which support the observations made in [Wang and Kilmartin. 2014] concerning the level and forms of social linkages within and between regions. These regional characteristics are integrated into the $\mathrm{ABM}$ developed in the current work and the performance of an alternative regionally priced DPS is also presented in section 4. Other analysis during this initial phase indicated that subscriber calling patterns could be well modelled using standard probability distribution functions (PDF). For example, Fig. 3 shows a lognormal fit to the subscriber call probability in Uganda when the average discount in the range of 50\%-60\%, similar to that suggested in [Seshadri et al. 2008]. The structure of social linkages amongst the subscriber base was also examined with Fig. 5 showing the subscriber degree distribution on a log-log scale. The result suggests that the degree distribution follows a power-law like distribution which 
would indicate that the underlying network is a scale-free network [Albert and Barabási. 2002].

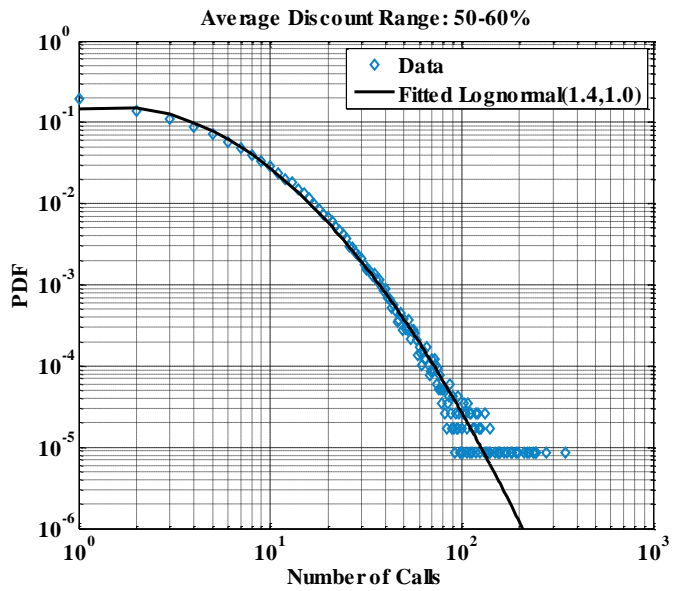

Fig. 3. Lognormal fit to the distribution of call attempts
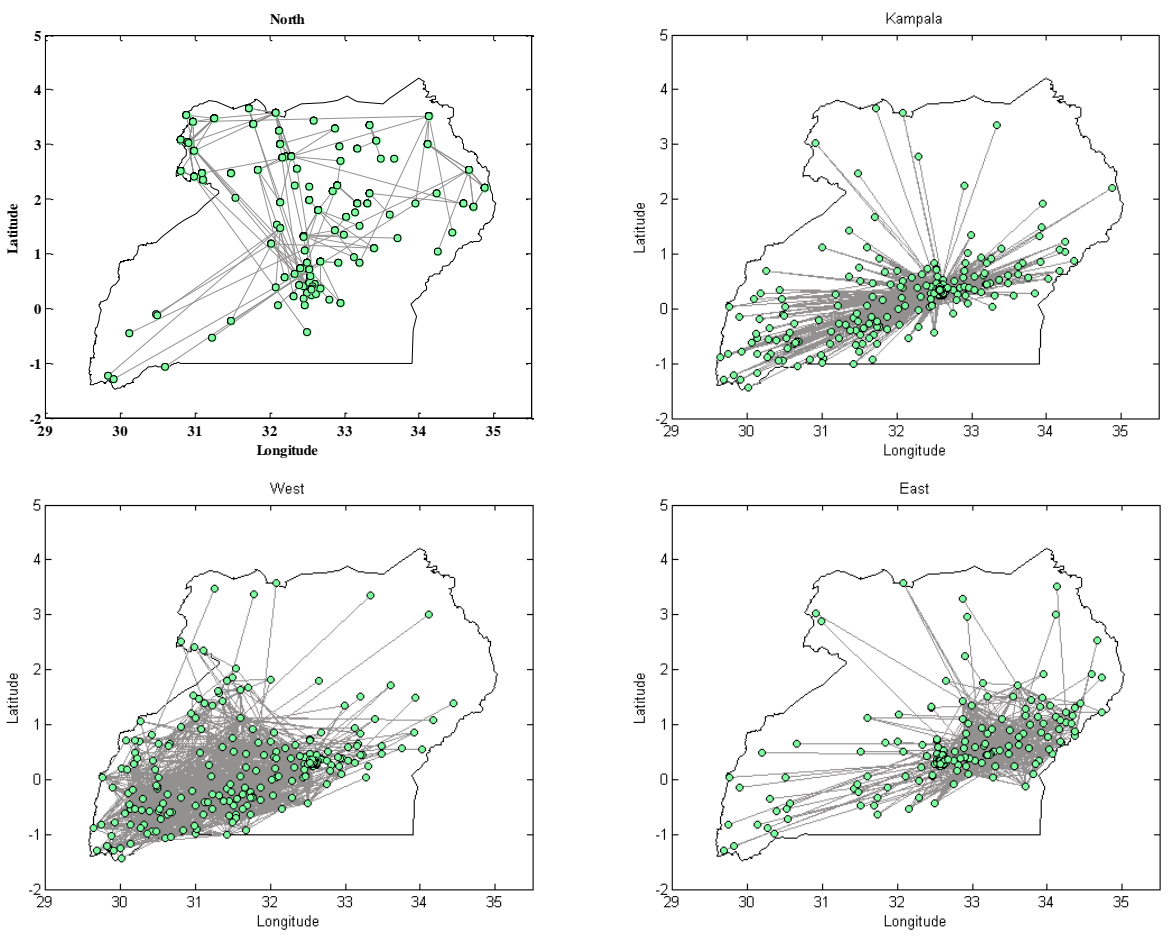

Fig. 4. Undirected weighted Mobile Call Graph (MCG) 
Investigating the Revenue Generation Impact of Dynamic Pricing Algorithms for Mobile Voice Services $1: 11$

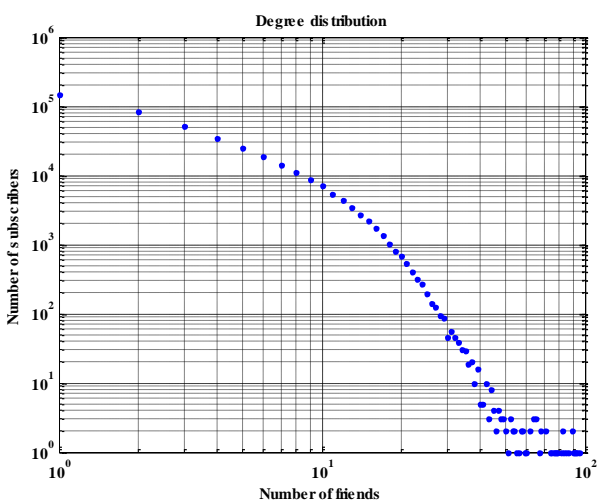

Fig. 5. Degree distribution on a log-log scale

\subsection{Structure of the Agent Based Model}

In ABM, agents are autonomous decision-making entities with diverse characteristics. Therefore, in our simulation of a mobile telephony network, the agents represent the individual subscribers and the agent's characteristics should simulate the real subscribers' calling, mobility and social behavior. In order to develop a realistic ABM, the behavior of the agents must replicate that of the real subscribers in terms of initiating voice calls at different time and locations to their social connections, in a manner which may be dependent on the DPS discount on offer at a given time. Therefore, the agent behavior contains three major sub-models:

(1) Call attempt model (when they call).

(2) Subscriber mobility model (where they call from).

(3) Subscriber social linkage \network model (who they call).

In order to produce a simulated behaviour which is similar to that of the real subscribers, each of these sub-models was designed based on analysing the real subscriber behaviour hidden in the CDR dataset. The following sections provide details of the design and operation of each of these agent sub-models of the overall model.

3.2.1. Call Attempt Model. The call attempt model is used to generate a probability of the agent "making a call" at each moment in time during a simulated day. In addition, this sub-model is also responsible for generating the duration of calls which the agent makes.

The call attempt probability will have two underlying drivers which mimic the fact that some calls in a DPS will be driven by the fact that a discount is on offer, whilst other calls are not discount driven and will be initiated regardless of the discount on offer from the DPS. Such a "non-discount" call might for example be related to the subscribers' employment or some urgent call which a subscriber would make no matter how much or little discount is offered. Alternatively, we also assume that there are some subscribers who are particularly sensitive to the discount offered to them and therefore their calling behavior may change based on the tariff that they are offered in real time. We will refer to calls such as this which are driven by the discount on offer from the DPS as a "discount" call. Hence, since most subscribers should exhibit a mixture of these two types of behavior, our model for the subscriber 
calling probability, containing both of these behaviors, factors the call probability as the probability that a user will make a call independent of the discount, $p_{n d}$, (which reflects the probability changing diurnally) and the probability that they will make a call influenced by the discount, $p_{n d}$ and the time of day, i.e. $p_{n d}, p_{d}$ as equation (1):

$$
p_{c_{i}}=\alpha_{i} P_{n d, i}+\left(1-\alpha_{i}\right) P_{n d, i} P_{d, i}
$$

where $\alpha$ is a constant factor to adjust the influence of discount on the calling behavior of subscribers, $P_{n d}$ is a probability distribution function for the "nondiscount" calls and $P_{d}$ is a probability distribution function for the discount driver calls.

The probability distribution function which we used for modelling $P_{\text {non_discount }}$ was determined by considering the calling behavior of subscribers in the CDR dataset when very little discount was offered by the DPS. For each subscriber, we used a kernel density estimation function [Bowman and Azzalini. 1997] as a fit to the subscriber's calling histogram during the day. Fig. 6 illustrate examples of this PDF for three subscribers as computed from the CDR data. When executing a simulation of a day's calling activity by the ABM, the $P_{\text {non_discount }}$ associated with an agent was simply sampled from the fitted PDFs for the real subscribers from the CDR data set.

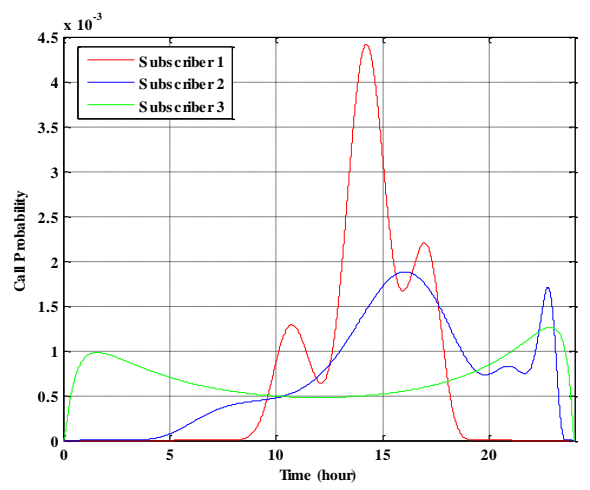

Fig. 6. Subscriber calling behavior

The second component in (1) relates to calls which are driven by the discount on offer from the DPS at a given time. In order to model the subscribers calling demand based on the dynamically varying tariff, we use a discount-demand function (2) which was proposed by Fitkov-Norris [Fitkov-Norris and Khanifar. 2000] to quantify the subscribers' response to the tariff offered.

$$
p_{d}=A e^{\lambda_{i} y}
$$

In (2), $y$ is the real time calling tariff (which is dependent on the offered discount) and $A$ is a demand constant shift with defaults to $1, \lambda_{\mathrm{i}}$ is the user demand parameter for user \agent $i$, as the discount demand function is an exponential, we use the conjugate prior to model the distribution individual users for $\lambda_{\mathrm{i}}$. The conjugate prior for an exponential is a Gamma distribution with parameters $a, b$. Thus, for a given agent $\lambda_{\mathrm{i}} \sim \Gamma(a, b)$. Fig. 7 illustrates an example of the resultant distribution for a subscriber's calling demand based on price. 
Investigating the Revenue Generation Impact of Dynamic Pricing Algorithms for Mobile Voice Services $1: 13$

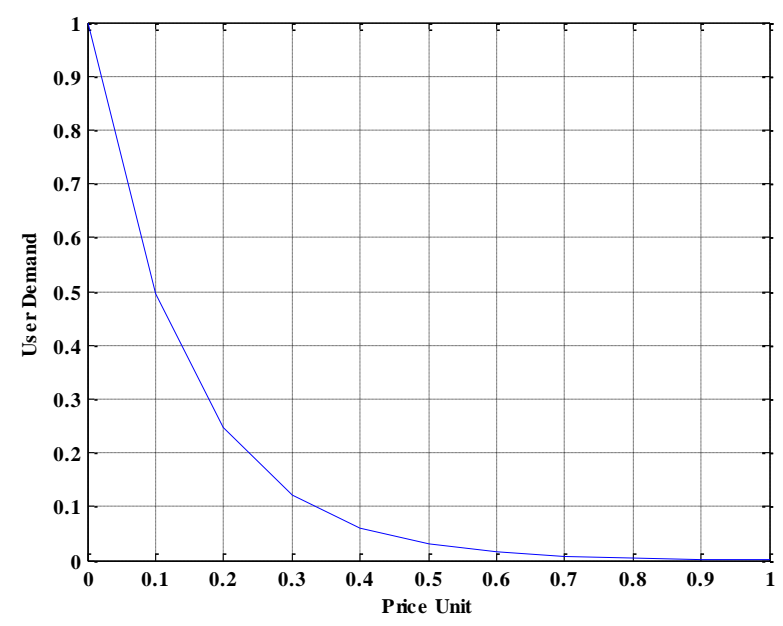

Fig. 7. Distribution of subscriber effect price on demand, $\boldsymbol{\lambda}_{\mathbf{i}}=\mathbf{7}$

The final element of the call attempt model relates to the duration of simulated calls. Unfortunately, no data was available in the CDR dataset relating to call durations and its dependency on discount offered for real calls made on the Ugandan network. As a result, and given the absence of any significant work in the literature that has examined this issue, we reverted initially to a model for call duration (in a static tariffing environment) proposed by Pattavina and Parini [Pattavina and Parini. 2005] whereby the random call duration was sampled from a lognormal distribution with $\mu=3.758$ and $\sigma=1.129$ (equivalent to a mean call duration of approximately 81 seconds), as illustrated in Fig. 8. However, this model was then modified on the assumption that subscribers will tend to elongate the call duration of discounted calls when the discounting factor offered by the DPS is large. The resultant model (3) is used to alter the average call holding time (and hence the value $\mu$ in the lognormal distribution) in an exponential fashion which increases with square of discount.

$$
\tau(y)=\tau_{0} \cdot e^{k(p)^{2}}
$$

where $\tau_{0}$ is the average call duration generated from the lognormal distribution. The value $k$ is a constant factor which can be used to adjust the impact of the discount multiplier effect. In this work, we utilized a value of $k=0.75$ as this resulted in a potential doubling in the average call holding time in scenarios when a very large discounts were offered, as illustrated in Fig. 9. 


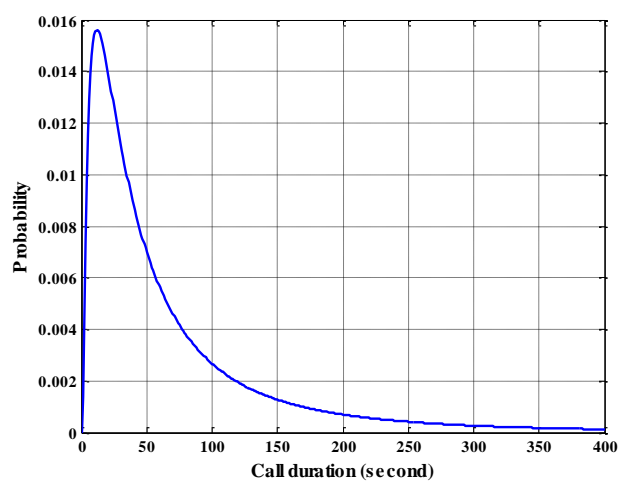

Fig. 8. Distribution of call duration

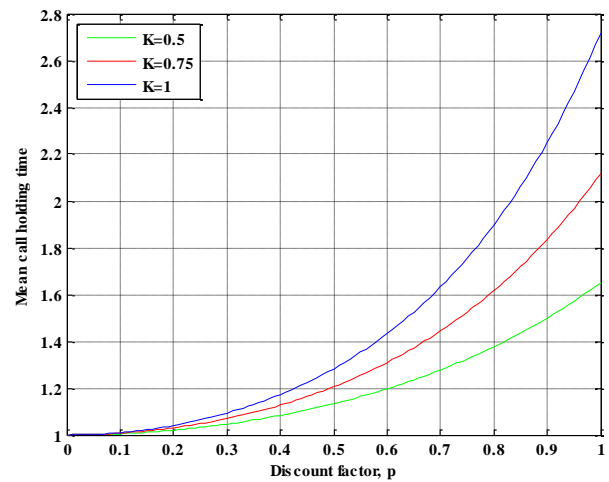

Fig. 9. Mean call holding time

3.2.2. Subscriber Mobility Model. The ABM contains a subscriber mobility model in order to simulate the actual behavior of subscribers in terms of their physical location in the network throughout the day and movement patterns between cells. The inclusion of this module within the model is important as the discount on offer to subscribers (and hence the revenue generated for the network operator) is dependent on the serving cell when the call attempt is made. In order to develop this model, we randomly selected 1000 subscribers from the CDR data set who made fewer than 50 calls and visited fewer than 20 cells per day. The location (i.e. cell site) and time of calls made by these subscribers were used to generate a two dimensional histogram (with bins based on time of day and cell site). We then applied a multivariate kernel density estimation [Ihler. 2007] algorithm to this histogram in order to estimate the probability of subscribers' calling locations at each moment during 2 hour intervals throughout the day. An example of one of the fitted PDFs is shown in Fig. 10, representing the PDF for subscribers calling locations at 10:00 am. 
Investigating the Revenue Generation Impact of Dynamic Pricing Algorithms for Mobile Voice Services $1: 15$

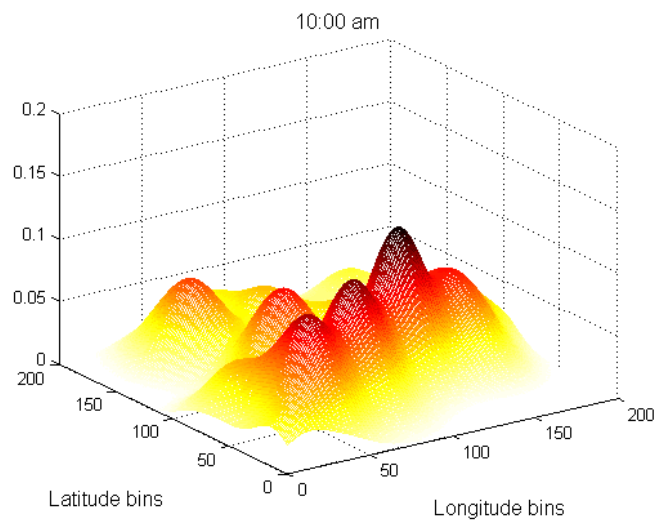

Fig. 10. Estimation of subscriber calling location at 10:00 am

(Longitude and Latitude divided into 200 bins)

3.2.3. Subscriber Social Linkage Network Model. The inclusion of a model of social connections between subscribers was important in order to accurately simulate the likely calling parties (and their geographic locations) in each simulated call between agents. In addition, in section 4 we discuss a number of alternative dynamic pricing strategies, a number of which are based around how often and who a subscriber is calling. For these reasons, the development of a suitable model for the social networks within the real subscriber base was important in the ABM. In order to develop a suitable model, we once again first look at the behavior present in the CDR data set. In this data set, we equate social interaction \linkages between subscribers as being reflected in the number of calls made between each other. Using a simple threshold on the number of inter-subscriber calls in the CDR data set as an indicator of a social linkage, we first examined the degree distribution of resultant social network. This degree distribution, as shown in Fig. 5, appears to follow a power-law distribution and, hence, there is evidence that the underlying social network is scalefree network [Barabási. 2009] properties. As a result, a Barabàsi Albert (BA) model was selected as the means of simulating an equivalent un-weighted scale-free social network [Albert and Barabási. 2002] for use within the $\mathrm{ABM}^{1}$. When simulating subscriber behavior, the ABM selects a potential called subscriber for each call based on the calling subscriber's contact list. However, the call probability to each contact is not uniform; individuals exhibit a large variability in the frequency of calls to their contacts. Therefore, we add a weight to the network for each subscriber connection. The weight is determined from the distribution of the number of calls to each of the subscriber's contacts. Based on the aggregated actual data, a Geometric distribution

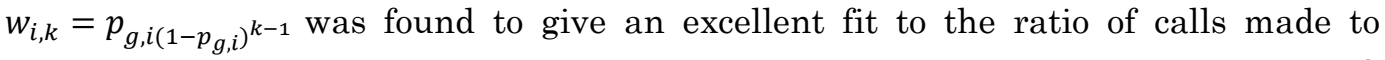
contacts. We use the value $w_{i, k}$ as a weight for the connections, where $i$ is the $i^{t h}$ agent and $\mathrm{k}$ is the $k^{\text {th }}$ contact ordered by popularity. To account for individual differences, we used the conjugate prior for a Geometric distribution, namely the Beta distribution with parameters $\alpha, \beta$; thus $p_{g, i} \sim \operatorname{Beta}(\alpha, \beta)$. The geometric distribution parameter (p) is obtained from a Beta distribution given a prior $\operatorname{Beta}(\alpha, \beta)$. These two parameters were estimated based on the distribution of the $p_{g}$ value for all users over the 19 days of data in the CDRs, as illustrated in Fig. 11

\footnotetext{
${ }^{1}$ We acknowledge that move complex models maybe of interest here but we leave this aspect to future research.
} 
(where values of $\alpha=2.3854$ and $\beta=4.6924$ were used). Fig. 12 also shows an example of this geometric fit for one particular subscriber in the CDR data.

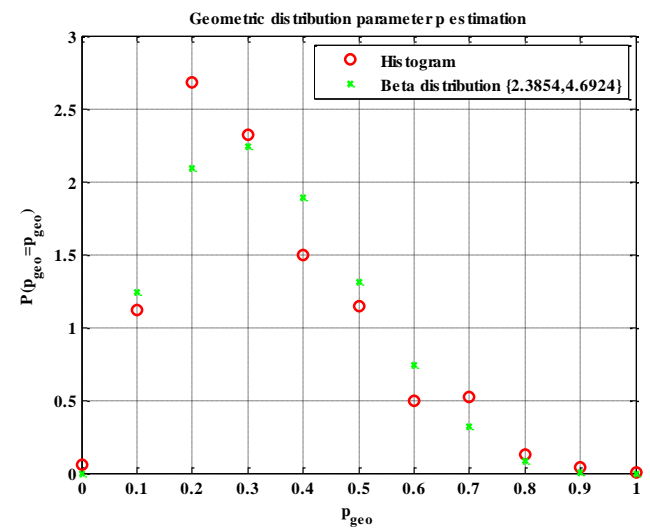

Fig. 11. Histogram of aggregated $\boldsymbol{p}_{g}, \forall_{i}$

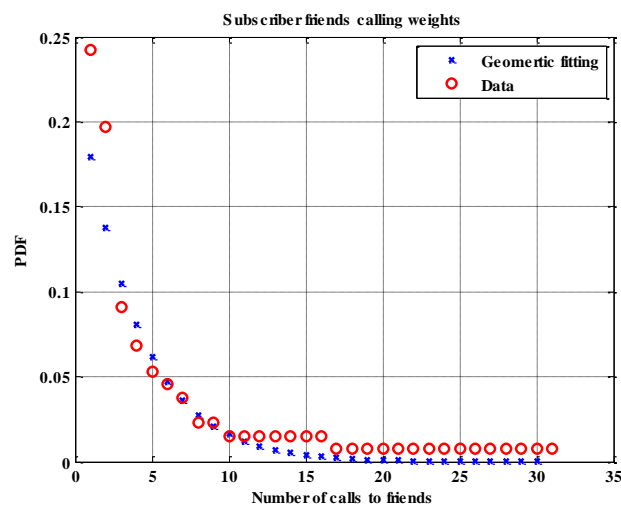

Fig. 12. Geometric distribution fitting for call frequency $\boldsymbol{p}_{g, i}=\mathbf{0 . 2}$ in this case

\section{DYNAMIC PRICING ALGORITHMS}

One of the primary motivators for the deployment of dynamic pricing in a mobile network is to allow the network operator to maximize the revenue generated through subscriber usage of their service. Having described in the process by which we designed the ABM in section III, this section of the paper will describe a number of alternative dynamic pricing algorithms whose revenue generating capabilities have been estimated using the developed ABM.

\subsection{Load Based Dynamic Pricing}

The initial pricing algorithm which was investigated using the ABM was one which modelled the algorithm used in the real mobile network from which the CDR data set was gathered. This pricing algorithm operated by offering a discount to a caller which was a function of the cell utilization factor at the time at which the call was made. An analysis of the call records in the data set, as shown in Fig. 13, resulted in a mapping between a cell utilization factor and the discount offered to the caller which was used when modelling this pricing algorithm. One challenge however which was encountered in terms of the implementation of this algorithm within the ABM was the issue of linking the cell utilization factor to the (channel) capacity of individual cells within the model. Whilst in the real network, there would likely be significant differences in the cell capacity in different cells in the network, it was decided that 
within the ABM framework, a cell's capacity would be modelled using a per call cell utilization change factor. In short, this would be the amount by which the cell utilization factor for a cell would increase when a new call was made in that cell (up to a maximum utilization factor of 1). Fig. 14 illustrates how this factor was calculated for each cell (using an analysis of the call records from the data set). The figure shows, for a particular cell in the real mobile network, how a linear approximation (whose slope of 0.043 is the per call cell utilization change factor) was fitted to data points plotted from the call records ${ }^{2}$.

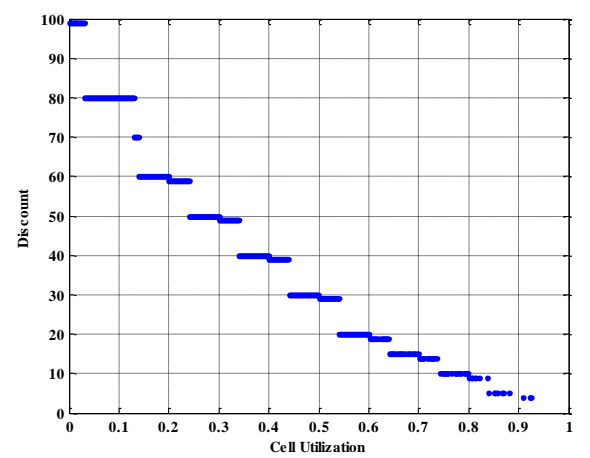

Fig. 13. The relationship between offered discount and cell utilization

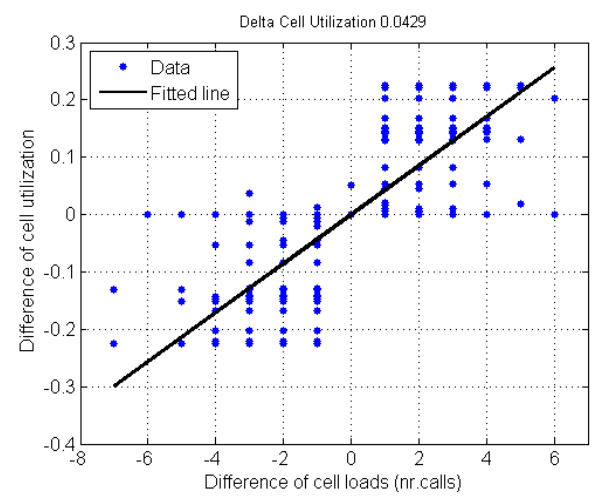

Fig. 14. The estimation of delta cell utilization

One issue which was quickly identified during initial simulations of this form of discounting algorithm related to the impact which this algorithm in particular had on revenue related to call attempts made very low load periods (i.e. in the middle of the night). During these hours, the cell utilization factor on most (if not all cells) was very low simply because there was little or no demand from subscribers to make calls at these times (e.g. between $2 \mathrm{AM}$ and 6AM). However, because the cell utilization factor would be very low at these times, the associated discount on offer would be very large. As a result, the revenue for what few calls were being made during this time period was being hugely diluted due to the un-necessarily large discount being offered. As a result, an initial variation on the cell load based discount algorithm was

\footnotetext{
${ }^{2}$ Note that for some imitated calls the measured utilization does not change as some ongoing calls end during the sampling period.
} 
to examine the use of a random discount in all cells throughout this "middle of the night" time period.

From a practical perspective, the deployment of this form of discounting algorithm would require significant infrastructure (e.g. CDR data warehousing and analysis systems) to be deployed to allow access to traffic load information (whether real time or averaged over a number of recent days) for all cells in the network. Clearly the scalability of such an approach in very large networks with potentially tens of thousands of cells would be practically and commercially quite challenging.

\subsection{Random Dynamic Pricing}

Whilst the use of a real time load based pricing algorithm has some attractive features (e.g. encouraging and discouraging service use during low and high load periods, respectively), there are significant practical and cost challenges in its deployment given the need to measure and process potentially large volumes of data concerning the loads in individual cells. An alternative dynamic tariffing strategy which addresses these challenges to some degree would be to utilize a random pricing algorithm. In practice this would result in the subscriber being offered a discount whose value would be drawn from some form of probability distribution function (e.g. discrete uniform distribution over the range of discount values. However, there still exists a spectrum of variants on this form of discounting which need to be considered. At one extreme is the scenario where a single PDF is used for all cells in the network throughout the complete day. While this approach would be quite attractive from an Operations and Maintenance (O\&M) perspective for the network operator, it is highly unlikely to result in a maximization of revenue generated from service usage. At the opposite extreme of the spectrum would be a deployment where each cell in the network uses its own PDF and that this PDF is changed regularly (e.g. every 15 minutes) throughout the day. Intuitively this form of a deployment is far more likely to result in higher revenue for the operator but its deployment would be extremely challenging. Such an approach would require significant and ongoing processing of "per-cell" revenue generation to determine the optimal PDF for each cell in the network (which could be of the order of tens of thousands in larger networks) and in each "time slot" during the day.

Hence, in this study, we selected an intermediate solution to these two extremes in order to investigate the potential of this form of discounting algorithm. The proposed algorithm was based on the utilization of only two time periods during the day (i.e. "off-peak" and "on-peak") and cells were grouped into four groups (with each region applying its own PDF applied to all cells in that region). The four cell groupings were based on the regional classification of cells (i.e. Northern region, Eastern region, Western \Central region and Kampala region) since our previous work [Wang and Kilmartin. 2014] identifying significant homogeneity in subscriber behavior within these regions. This approach would also be a very reasonable approach for the practical management of such a deployment given that a network O\&M center would only have to monitor, configure and control the performance of the system (and its revenue generation characteristics) using the manageable combination of four regions and two time periods.

\subsection{Subscriber Centric Dynamic Pricing}

The final form of dynamic pricing algorithm which was investigated in this work was one which was subscriber centric, in the sense of offer high value subscribers of the network enhanced discounts. The algorithm would operate by offering all subscribers 
Investigating the Revenue Generation Impact of Dynamic Pricing Algorithms for Mobile Voice Services $1: 19$

to the service a baseline discount (in the form of a random discount implementing a relatively low mean discount value similar to that outlined in section 4.2. However, the approach requires a certain percentage of subscribers who exhibited some key characteristics to be offered a significantly enhanced discount (again implemented in the form of a random discount but with a far more substantial mean discount value). Two different methodologies for identifying these high value subscribers were investigated in this work namely, (i) the subscribers with the largest average number of call attempts per day and (ii) the subscribers with the largest number of contacts in their social network structure (i.e. largest degree value). This form of discounting algorithm would likely be quite attractive to a network operator in terms of the practicalities for deployment. Since the vast majority of subscribers simply receive the same (low) random discount, there is little need for significant high volume data processing in terms of discount calculation (for example as is required in the load based discounting algorithm). The process of selecting the key subscribers would require significant analysis of subscribers' calling patterns but this could be done in an off-line manner (using short term historical analysis of CDRs) at some regular time interval. The nature of the algorithm could also introduce from a service marketing perspective the opportunity for gamification in an attempt to encourage subscribers who are not in receipt of the larger discounts to increase their service usage pattern.

We further investigate this last phenomenon within our model by inclusion of an additional component to the call attempt model introduced in section 3.2.1. When investigating this form of subscriber centric discounting, we include a component whereby subscribers who receive calls from a subscriber in receipt of the large (high value subscriber) discount levels may increase their call generation rate. The role of this enhancement is to model the likely effect by which the calling party might inform that the called party that they were in receipt of enhanced discounts because of their high value status. This, in turn, in some instances could influence the calling party to make more calls in an attempt to also achieve this status in the network of subscribers. The modelling approach which we utilize to simulate this effect is based on an adaptation of the epidemic spreading model proposed by Barrett et al. [Barrett et al. 2008], and outlined in (4).

$$
\Delta p_{d, i}=1-e^{\left(\sum_{r \in R} \ln \left(1-k s_{i}\right)\right)}
$$

where $\Delta P_{i}$ is an increase in the discount sensitive calling probability of subscriber $i, s_{i}$ is reflects the susceptibility of normal subscriber $i$ to "advertising" encouraging them to increase their use of the service (i.e. in the form of the high value subscribers calling them and telling them that they can get more discount by making more calls), $k$ is a constant which reflects the strength of high value subscribers as "advertisers" of the service, $R$ is the set of "advertisers" (i.e. "advertising" subscribers) and $r$ is the individual "advertiser".

In our simulation, this equation was calculated at the end of each simulated day in order to determine the increase of $\Delta p_{d, i}$ for next simulated day of network operation. Similar to epidemic spreading in the social networks as described in [Barrett, Bisset, Eubank, Feng and Marathe. 2008], we also assume that these normal subscribers will continue to increase their calling probability if they keep getting the calls from 
high value subscribers each day. On the contrary, they will reduce their calling probability by a factor of $2 / 3$ if they do not receive any calls during the preceding day from high value subscribers. Hence, the increase in the subscriber calling for each agent was computed using (5):

$$
\gamma_{i}^{t}=\left\{\begin{array}{c}
\Delta p_{d, i}^{t-1}+\Delta p_{d, i}^{t}\left(1+\Delta p_{d, i}^{t-1}\right), \\
\frac{2}{3} \Delta p_{d, i}^{t-1}, \text { othereived call }(s) \text { from high value subscribers }
\end{array}\right.
$$

Where we now index $p_{d, i}$ by time where $\Delta \mathrm{p}_{\mathrm{d}, \mathrm{i}}^{\mathrm{t}-1}$ the previous day is. Therefore, the resultant discount sensitive probability $\left(p_{d, i}\right)$ determined in (2) is modified using (6) to provide $p_{d}^{*}$ which is used in the simulation.

$$
p_{d, i}^{*, t}=p_{d, i}\left(1+\gamma_{i}^{t}\right)
$$

\section{RESULTS}

This section of the paper firstly presents the results obtain during the process of tuning certain model parameters in order to achieve comparable results with the subscriber behavior observed in the underlying CDR data set used in this work. Subsequently, the results of a comparative study carried out using the ABM on the revenue generation performance of the various dynamic pricing algorithms which were introduced in Section 4 is presented. Fig. 15 provides an illustration of the $\mathrm{ABM}$ simulation process used in generating these results. In general, there are four major steps in this simulation:

(1) Agents initialization.

(2) Voice call simulation.

(3) Discount offering and.

(4) Cell utilization updating.

Unless otherwise stated, all results were generated after multiple simulation runs of the ABM using a given configuration. Each of these simulation runs were based on a simulation scenario consisting of 1000 simulated agents operating in the simulated DPS environment for a period of 19 days (as per the underlying CDRs). 
Investigating the Revenue Generation Impact of Dynamic Pricing Algorithms for Mobile Voice Services $1: 21$

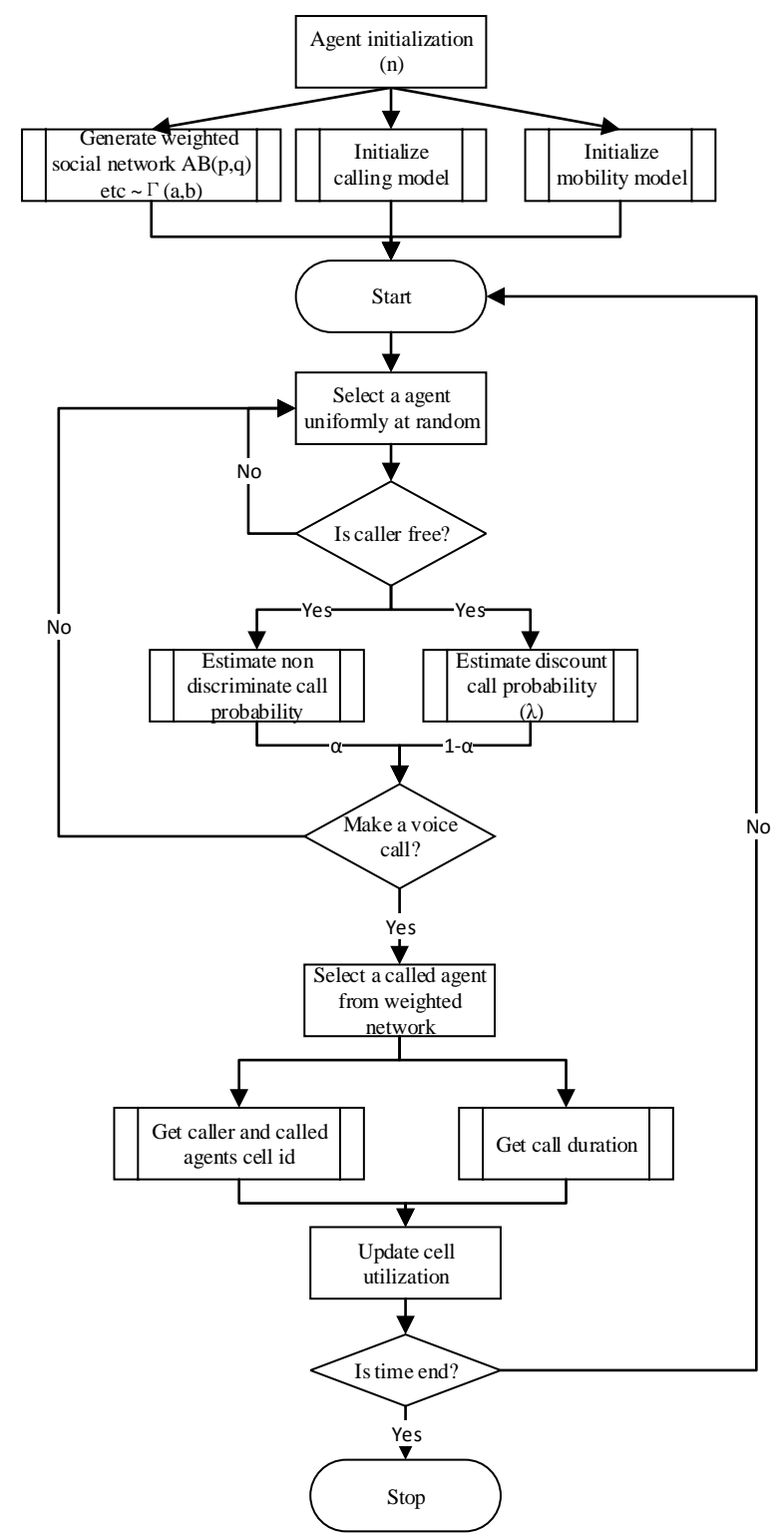

Fig. 15. ABM Simulation

\subsection{Model Parameter Tuning}

One of the key parameters in the model which could not directly be estimated from analysis of the CDRs is the $\alpha$ value in the call attempt model as shown in (1). This parameter effectively controls the relative number of call attempts which are discount driven (as distinct to calls which would occur regardless of the discount level on offer). In order to determine a reasonable value for this parameter, simulations were carried out using a series of values for $\alpha$ ranging from 0 (i.e. all subscriber calls are motivated by the offered discount) to 1 (i.e. no subscriber calls are motivated by the offered discount). Fig. 16 shows a comparison between the total number of calls (in bins of 15 minute duration) per simulated day generated by the ABM and the 
equivalent data calculated from an analysis of the CDR data set, for settings of $\alpha=$ $0.1, \alpha=0.5$ and $\alpha=0.8$ respectively. These clearly show that the behavior of the model with a setting of $\alpha=0.8$ is qualitatively comparable to the behavior observed for the real subscribers. Furthermore, Fig. 17 contains a plot of a normalized root mean squared error (RMSE) between the simulated and real call rate (derived from the CDRs) for the range of investigated a values. As a result, a value of $\alpha=0.8$, which resulted in a normalized RMSE value of $\approx 0.1$, was selected as offering a good match with the behavior present in the CDRs (whilst not having a reasonable dependence on offered discount within the call attempt model).

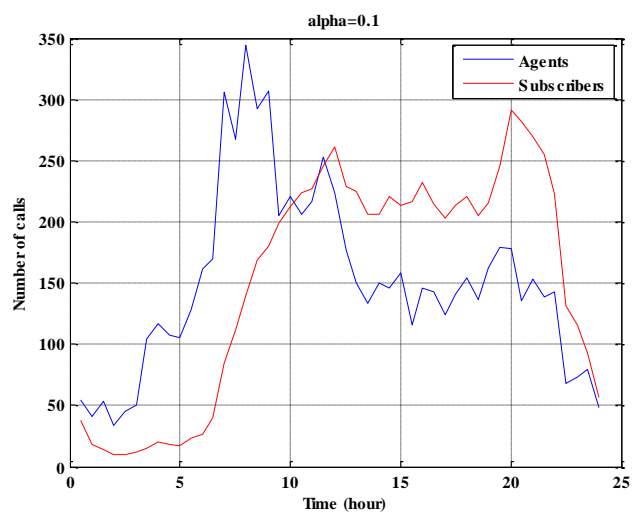

(a)

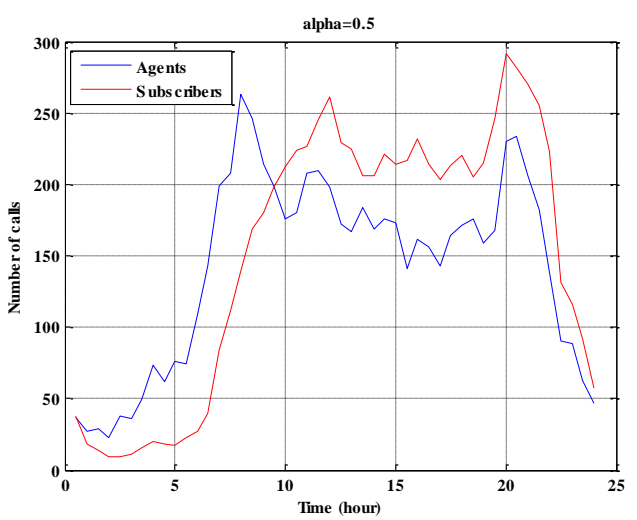

(b) 
Investigating the Revenue Generation Impact of Dynamic Pricing Algorithms for Mobile Voice Services $1: 23$

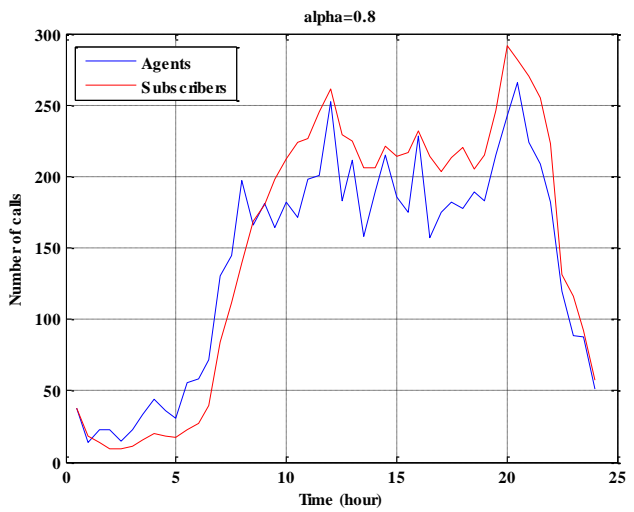

(c)

Fig. 16. The simulation results of the total number of calls over a day based on different influential of discount factor $\boldsymbol{\alpha}$. (a) $\alpha=0.1$. (b) $\alpha=0.5$. (c) $\alpha=0.8$

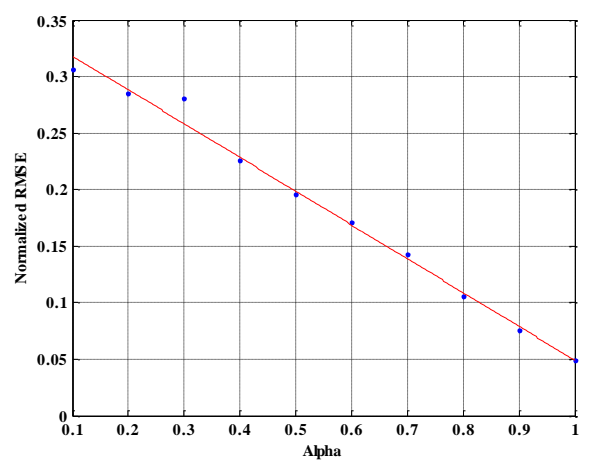

Fig. 17. Normalized root mean square error

The user demand factor ( $\lambda$ in equation (2)) is sampled from a Gamma distribution with parameters $a, b$ as noted in section 3.2.1. The parameters of this distribution were set to $a=8$ and $b=0.7$ such that a larger proportion of agents in the simulation would more likely exhibit a behavior where discount motivated calls would only be made when larger rather than smaller discounts were offered. Fig. 18 provides an illustration of a set of resultant user demand versus discount curves generated from this distribution. In terms of the social network $\backslash$ linkage model, a BA network generator with its parameters were set to $p=0.2, q=0.3$ and $m=2$ was used to simulate the inter-subscriber network structure as discussed in section 3.2.3. 


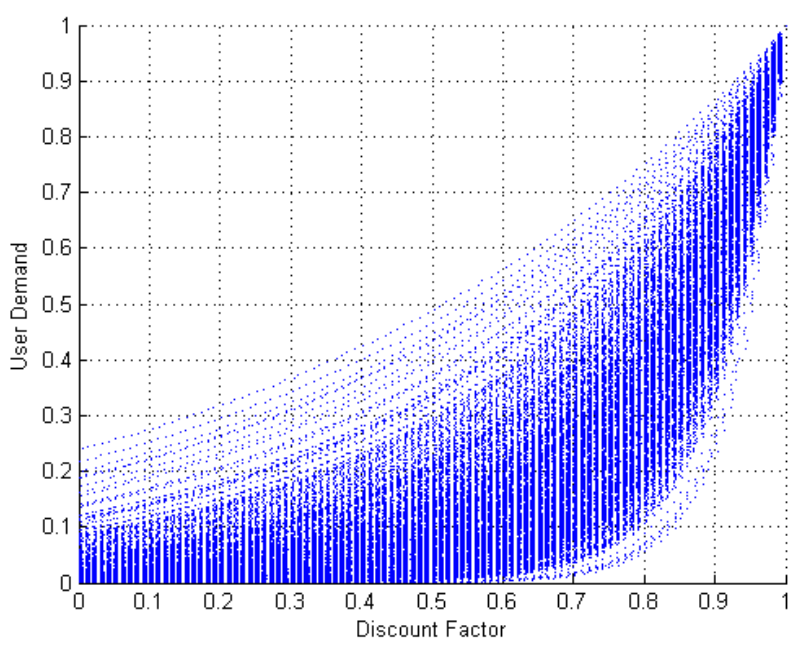

Fig. 18. Samples of the user demand function $\boldsymbol{\lambda}_{\boldsymbol{i}} \sim \boldsymbol{\Gamma}(\mathbf{8}, \mathbf{0 . 7})$

Utilizing these various parameter settings, Fig. 19 provides a more comprehensive comparison of the behavior of the ABM with the subscriber behavior exhibited in the CDRs. Fig. 19 (a) shows the average discount the subscribers obtained over a day (using the load based discounting algorithm in the ABM as was used in the real DPS deployment). Fig. 19 (b) illustrates the average cell utilization over a day and Fig. 19 (c) highlights the distribution of the number of calls per subscriber. The results show that the results obtain from the ABM simulation are quite close to the behavior observed from the real CDR data set across all three of these metrics.

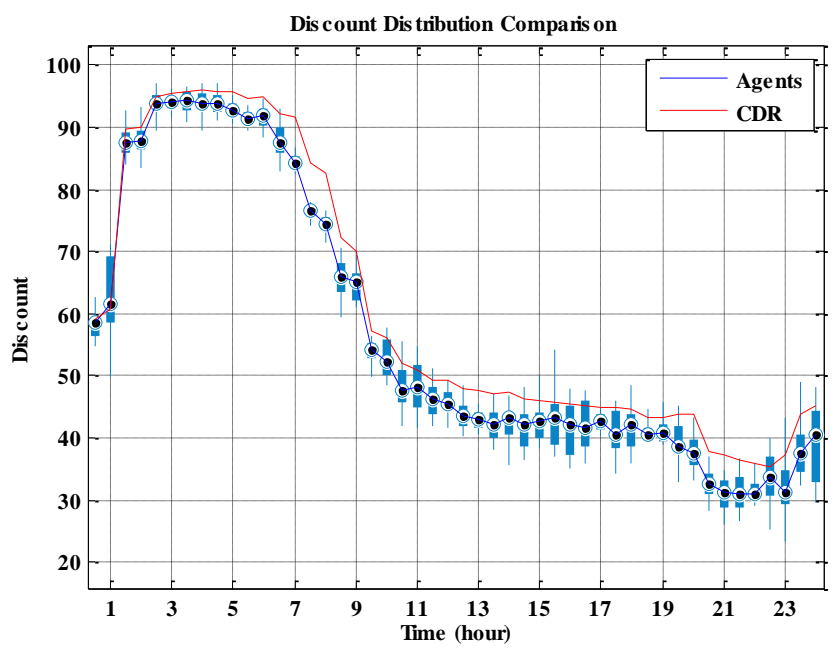

(a) 
Investigating the Revenue Generation Impact of Dynamic Pricing Algorithms for Mobile Voice Services $1: 25$

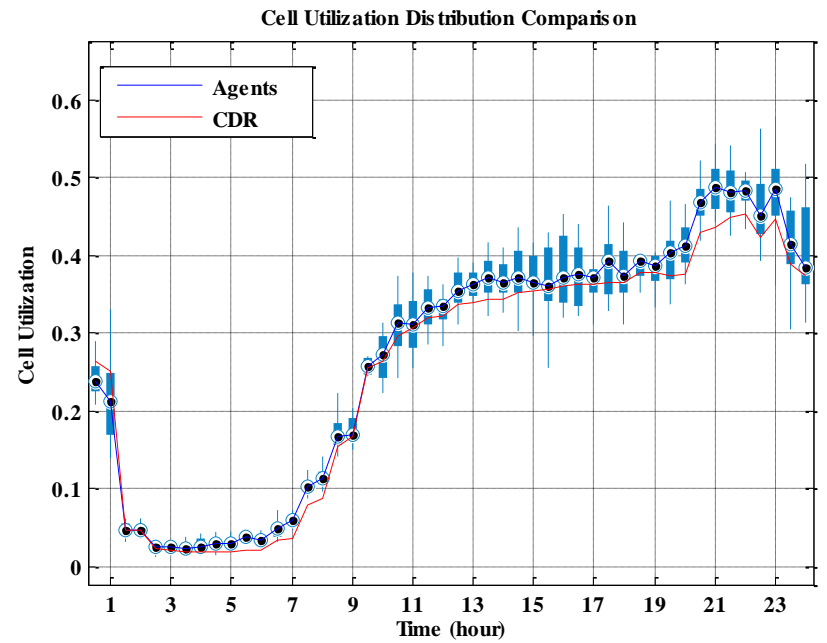

(b)

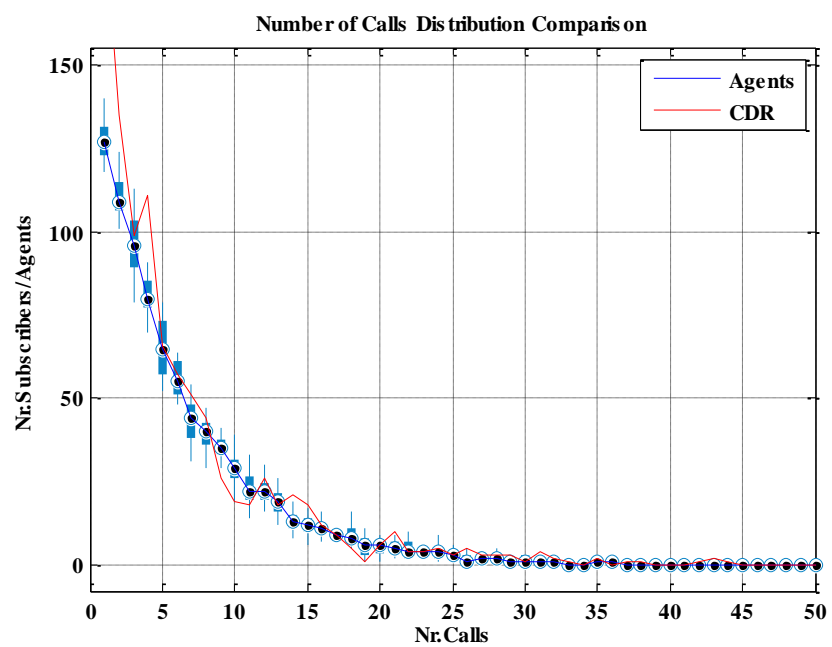

(c)

Fig. 19. Comparison between $\mathrm{ABM}$ simulation results and CDR data set. (a) discount distribution comparison. (b) cell utilization distribution comparison. (c) histogram of the number of calls distribution comparison.

\subsection{Off-Peak Discount Strategy}

As we have mentioned in section 4.1, a significant issue which was initially investigated related to the impact which discounting would have on revenue generation during the significant "off-peak" time period in the middle of night. As can be seen in the plot of call activity extracted from the CDR data set in Fig. 19, this period (when averaged across the real network) extends from approximately 2 AM and $6 \mathrm{AM}$. For marketing \commercial reasons, it is likely that some form of discounting strategy would have to be offered during this time period. There was no evidence present in the CDRs that offering very significant discounts (as was the case in the real network where the load based discounting algorithm offered huge 
discounts during this low load off peak time period) had any impact on encouraging significant call traffic during this time period. Hence we propose that a random discount be offered during this "off-peak" time period (regardless of what strategy would be utilized during the rest of the day when traffic is far more significant). In order to evaluate the impact on revenue of possible discount levels offered during this period, we used the ABM to simulate the scenario where a random discount drawn from a uniform distribution between $X-2 \%$ and $X+2 \%$ (where $X$ ranged from $2 \%$ to 98\%) was offered to "off-peak" calls. Fig. 20 illustrates the results of this simulation (after $\mathrm{N}=10$ simulation runs of the $\mathrm{ABM}$ ) with the normalized (relative to no discount being offered) revenue generated for the operator being plotted against various values of $\mathrm{X}$ (with a polynomial spline fitting). It is clear from these results that significant amounts of "nighttime" revenue losses would be incurred if (mean) discounts greater than $5 \%$ are offered during this time period. However, as noted in Fig. 20, the revenue generated during this "night-time" period is quite small (i.e. approximately $1.75 \%$ of overall daily revenue) and hence larger discounts might in practice be considered as a marketing "gambit". However, we have utilized a random discount with a mean value of $3 \%$ during the 2:00 am to 6:00 am time period in all subsequent investigations of dynamic pricing algorithms, in order to maximize revenue.

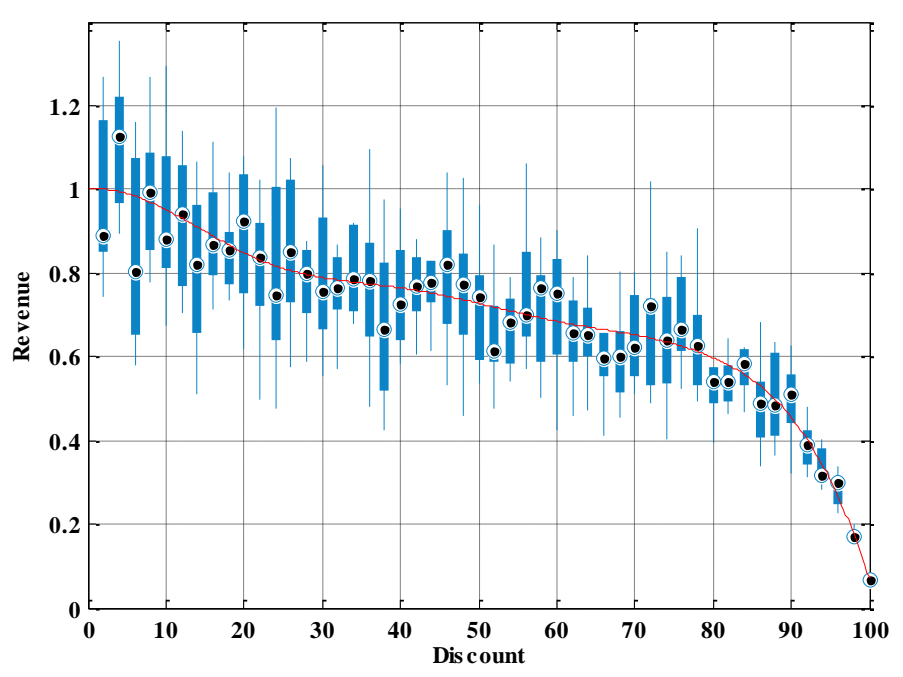

Fig. 20. The impact of revenue based on discounts (in the hours 2am-6am).

\subsection{Subscriber Centric Discounting Algorithm Operation}

Section 4.3 outlined a proposed paradigm for delivering a subscriber centric discounts. Using this approach we proposed to offer, during "daytime hours", a larger discount to a cohort of high value key subscribers (i.e. selected either based on node degree or call volume). In practice the size of the cohort of subscribers to which these larger discounts would be offered would likely be driven by marketing rather than technical forces. In our simulations, it was decided that we would investigate the case where $25 \%$ of the subscriber base were offered these enhanced discounts. We examined the discount impact on revenue for both the high value subscriber base and the remaining "normal" subscriber base. Initially, a uniform random discount with a mean value of $5 \%$ (with $2 \%$ standard deviation) was offered to the "normal" subscriber base with the (mean) discount offered to the key subscribers being varied 

$1: 27$

to investigate the impact on overall revenue. Fig. 21 (a) shows the revenue generated when the (mean) discount offered to the high value subscriber cohort had a mean values between $20 \%$ and $50 \%$. This graphs shows that the revenue reduces in a manner which is reasonably well modelled by a linear fitting for both the case where the high value cohort is selected based on call volume and for case where these subscribers are selected based on their connection degree. It also highlights that this decrease in revenue appears to be slightly less for the latter means of selecting the high value subscriber group compared to the former selection criterion.

In order to visualize the impact which the discount offered to the "normal" subscriber cohort has on revenue, a similar analysis was completed except this time with the discount offered to the high value subscriber group being fixed (at 25\%). Fig. 21 (b) illustrates the results of this simulation and highlights the need to keep the (mean) discount offered to this "normal" subscriber small in order to avoid significant revenue losses. Clearly the actually (mean) discounts offered to these two subscriber groups in practice would have to be set at "reasonable" value for marketing and, likely, regulatory reasons and hence in subsequent comparisons, we have utilized mean discounts of $20 \%$ and $5 \%$ for the high value and "normal" subscriber groups, respectively.

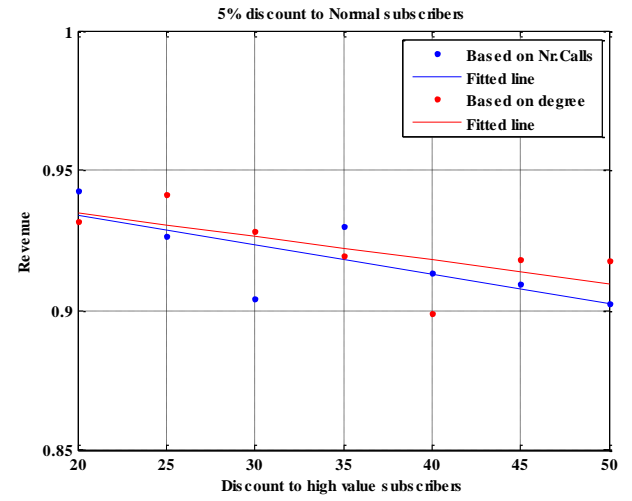

(a)

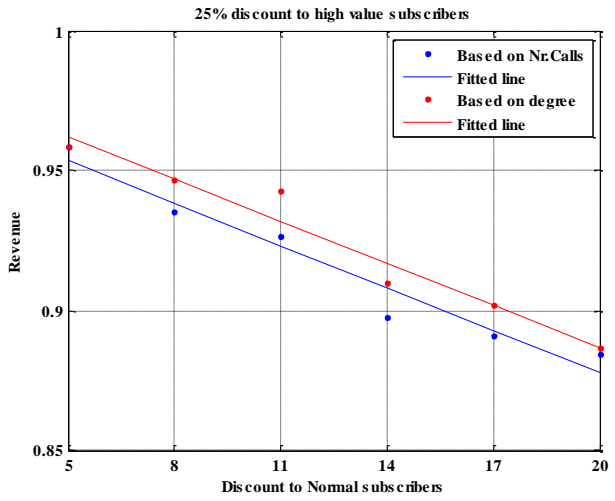

(b)

Fig. 21. (a) Impact of mean discount offered to "normal" subscribers on revenue (b) Impact of mean discount offered to high value subscribers on revenue.

5.3.1. Inclusion of Epidemic Spreading Model. Section 4.3 further outlined the inclusion of an epidemic spreading model between agents which could potentially result in an increase in the likelihood of discount sensitive calls being generated by individual agents. The impact of the inclusion of this behavior into the agent model was further investigated through simulation runs of the ABM. In an initial simulation, each agent was initialized with a $S_{i}$ value (from (4) ) which was drawn from a lognormal distribution with $\mu=0.6$ and $\sigma=0.05$. Fig. 22 present the average increase in discount motivated call generation probability $(\gamma)$ over a 50 day simulation run, for scenarios where high value subscribers are selected based on call volume and based on social connections (i.e. node degree). 


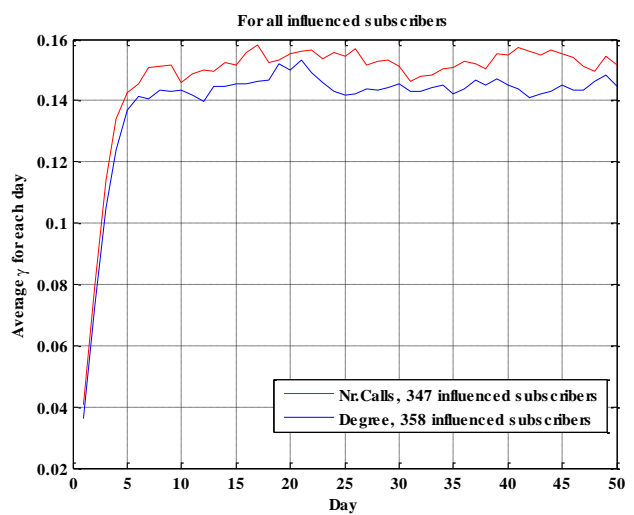

(a)

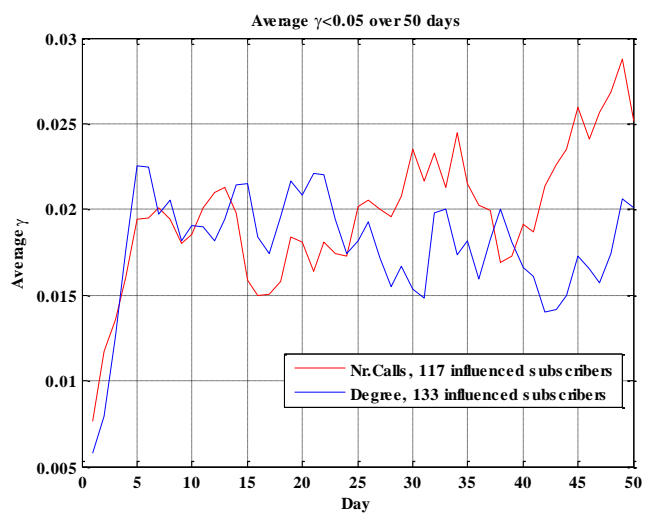

(b)

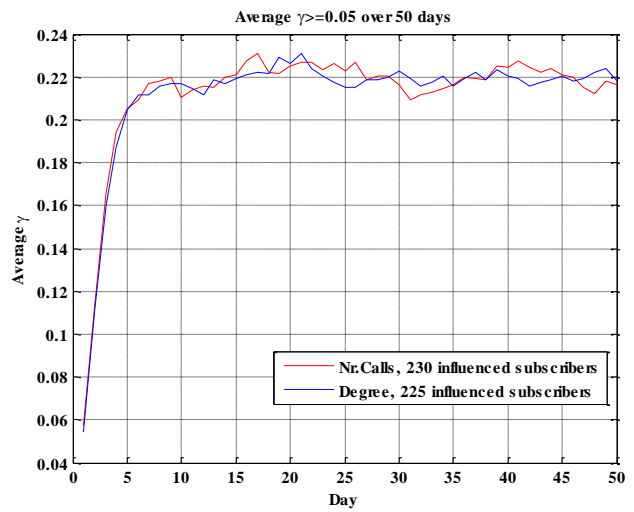

(c)

Fig. 22. Evolution of average $\gamma$ over the total of 50 days for (a) all subscribers (b) "mildly" influenced subscribers and (c) "significantly" influenced subscribers

The results indicate that approximately $45 \%$ of "normal" subscribers (i.e. 347 or 358 subscribers out of 750 "normal" agents) have experienced some level influence on the basis of calls received from the high value subscriber group during the simulation period of 50 days. By splitting in the influenced subscriber group into two based on the level of influence (using a threshold of 0.05 for the average $\gamma$ value, Fig. 22 (b) highlights that $37 \%$ of influenced subscribers were only "mildly" influence over the simulation period while Fig. 22 (c) illustrates the temporal evolution of the $\gamma$ value 

$1: 29$

for the remainder of the group who experienced more significant influence (i.e. these subscribers' discount sensitive probability of generating a discount sensitive call would have increased (on average) by $22 \%$ by the end of the 50 day simulation period). These results would suggest that this could result in significant changes taking place in the group of subscribers forming the high value group when this would be reviewed by the network operator at some regular interval. The impact of the inclusion of the epidemic model on the estimated revenue generated for the network operator was far less pronounced. In fact the revenue estimates from the ABM, indicates that inclusion of the epidemic model has a negligible effect on revenue (i.e. $0.53 \%$ more revenue compared to the non-epidemic model).

\subsection{Comparison of Dynamic Pricing Strategies}

In this section, we compare the overall revenue generation capabilities of the three types of dynamic pricing strategy that were introduced in section 4 . The analysis of the impact on revenue for different strategy was examined based on a regional approach (using the four geographic regions of Uganda, as discussed in section 2). For each region, we firstly investigated the operation of a random discount algorithm operating during "daytime" hours at a regional level. In this simulation, the revenue generated when subscribers were offered (mean) discounts at different levels between $0 \%$ and $100 \%$ was modelled. Subsequent simulations using the ABM focused on estimating revenue generated when (a) a load based discounting strategy (as outlined in section 4.1) was used and (b) when the subscriber centric discounting algorithm outlined in section 4.3 and 5.3, were active. Fig. 23 provides a graphical comparison of the impact on revenue of the discounting algorithms when simulated independently within the four geographical regions of the country.

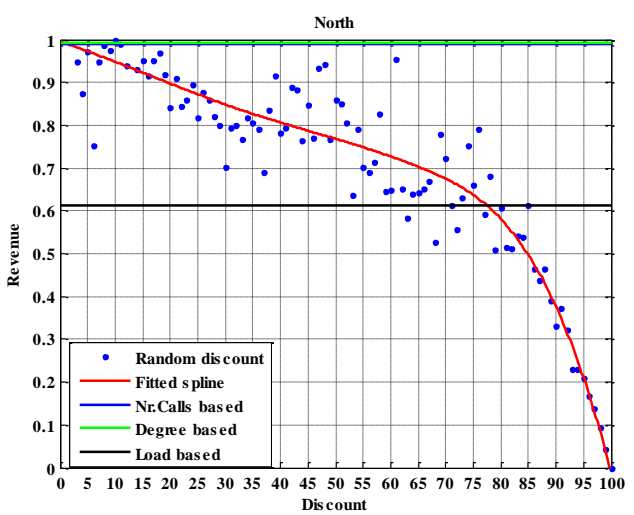

(a)

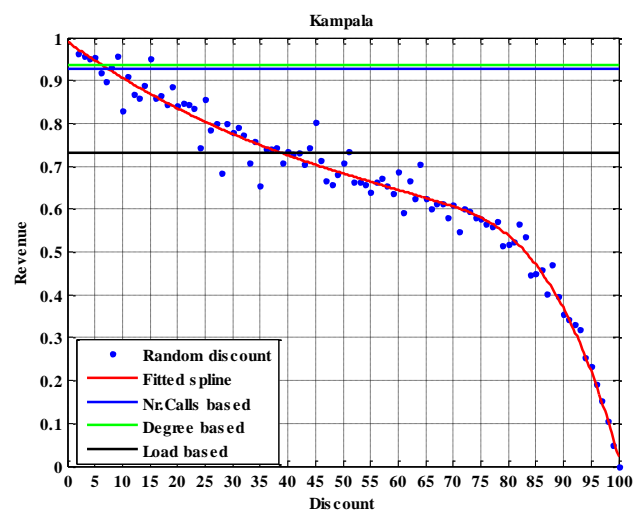

(b) 


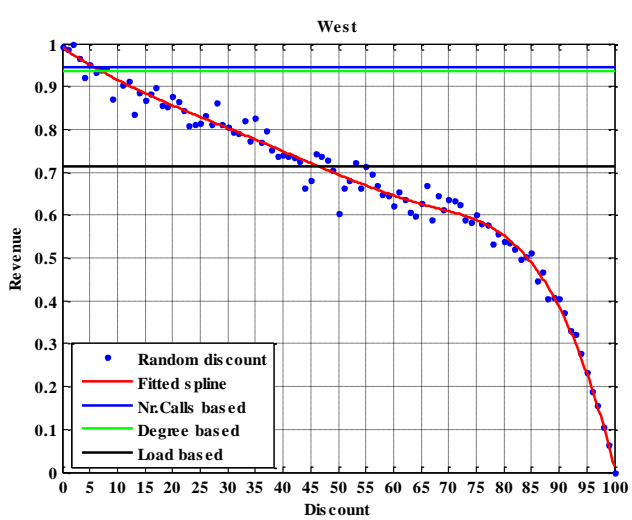

(c)

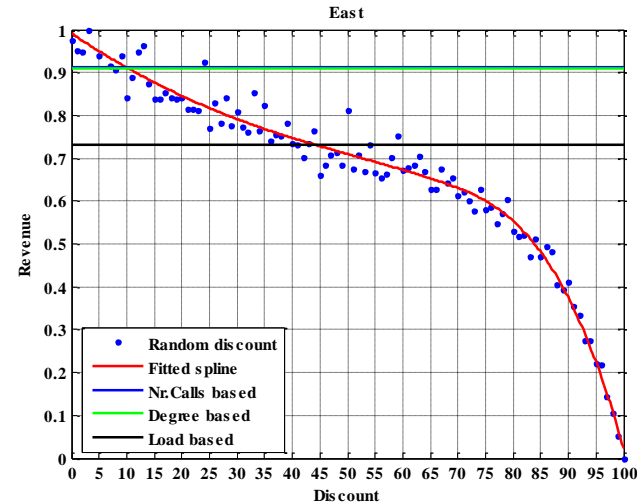

(d)

Fig. 23. Comparison of regional revenue generation

Table II provides a summary comparison of normalized (relative to the nondiscounted case) revenue when load based discounting and subscriber centric based discounting (using either call volume or subscriber social links as the means of selecting the high value subscriber cohort). In addition, the table also indicates the equivalent (mean) random discount which would have to be offered to achieve a similar level of revenue.

Table II. Comparis on of normalized revenue and equivalent mean random discount

\begin{tabular}{|l|c|c|c|c|c|c|}
\cline { 2 - 7 } \multicolumn{1}{c|}{} & \multicolumn{3}{|c|}{$\begin{array}{c}\text { Revenue normalised to non- } \\
\text { discounted case }\end{array}$} & \multicolumn{2}{c|}{ Equivalent mean random discount } \\
\hline $\begin{array}{c}\text { Region } \\
\text { North }\end{array}$ & $\begin{array}{c}\text { Load } \\
\text { Based } \\
\text { Discount }\end{array}$ & $\begin{array}{c}\text { Subscriber } \\
\text { Centric } \\
\text { (Call } \\
\text { Volume) }\end{array}$ & $\begin{array}{c}\text { Subscriber } \\
\text { Centric } \\
\text { (Degree) }\end{array}$ & $\begin{array}{c}\text { Load } \\
\text { Based } \\
\text { Discount }\end{array}$ & $\begin{array}{c}\text { Subscriber } \\
\text { Centric } \\
\text { (Call } \\
\text { Volume) }\end{array}$ & $\begin{array}{c}\text { Subscriber } \\
\text { Centric } \\
\text { (Degree) }\end{array}$ \\
\hline Kampala & 0.6122 & 0.9889 & 0.9934 & 78 & 3 & 2 \\
\hline West & 0.7325 & 0.9253 & 0.9357 & 39 & 9 & 7 \\
\hline East & 0.7128 & 0.9447 & 0.9355 & 48 & 7 & 8 \\
\hline \hline Country & 0.7307 & 0.9125 & 0.9106 & 45 & 11 & 11 \\
\hline
\end{tabular}

The relationship between the level of random discount offered and revenue generated, as shown in Fig. 23 appears to be very similar in three of the four geographical regions. However, the characteristics of this relationship for simulated North region of the country is somewhat different in that, initially, as the level of discount being offered is increased the fall in revenue is less pronounced. For example, in the North region, a random discount of approximately $65 \%$ would result in a normalized revenue value of 0.7 , whereas in the other three regions this would occur when discounts of only $45-50 \%$ were offered. Whilst in absolute terms, these all represent revenue loss for the operator (compared to the case of offering no discount), it does highlight the fact subscriber (agents) in the North region are more attracted to this form of tariff in that it encourages them to increase their level of usage of the service when large discounts are offered compared to the agents in the other regions. This would provide some evidence that random discounting may be more attractive in regions where subscribers are particularly price sensitive (as would likely be the case in the mostly rural and economically under-developed Northern region of Uganda). Clearly, as noted above, based on the simulation results such a pricing algorithm would still result in an absolute loss in revenue for the operator which would have to be addressed by other mechanisms. 
Investigating the Revenue Generation Impact of Dynamic Pricing Algorithms for Mobile Voice Services $1: 31$

Table II clearly illustrates that load based discounting results in very poor revenue generation for the operator. At a national level, the results such that an approximately $30 \%$ drop in network operator revenue would result were a discount of this nature to be deployed. When we examine the revenue at a regional level, it is clear that a pricing paradigm of this nature would perform particularly poorly in the North region of our simulation with a nearly $40 \%$ drop in revenue being observed. The root cause of this very poor regional performance is the small subscriber based present in this geographic region resulting in there being consistent over-capacity in the cell sites of this region. Hence, some significant adaptation of the load based algorithm would have to be undertaken due to the fact that, for the majority of the time, most cell sites are very lightly load (and, hence, unnecessarily offer large discounts).

In terms of maximizing revenue for the network operator, the results suggest that there are really only two sensible options. Firstly, operators could use random discount algorithms but with mean values which are very small. However, in practice this approach would not be feasible from a marketing perspective and unlikely to maintain customer satisfaction, given a likely expectation amongst subscribers of getting more than low single digit discounts. The second alternative is the deployment subscriber centric discounting. The simulation results indicate that such algorithms would result in less than $6 \%$ revenue loss to the operator. From a marketing and subscriber retention perspective, this form of algorithm is also far more palatable given that all subscribers would receive discounts and the high value subscriber group in particular would receive significant discounts. In addition, the "competitive" nature of the algorithm (in terms of which subscribers are selected for inclusion in the high value group) would likely be particularly attractive from a marketing perspective. A potential downside of this form of algorithm, particularly compared to random discounting, is the additional infrastructure (and associated operations and maintenance overhead) associated with the process of how very large volumes of CDRs are collated and processed in order to regularly update membership of the high value subscriber cohort.

It is clear from the simulation results in Fig. 23 that in absolute terms revenue is maximized when no discount is offered to subscribers. Also it is interesting to note that using the model which has been outlined there does not appear to be a secondary maximum in the revenue-versus-discount plots. This is noteworthy as it would suggest that there is no role to be played by optimization algorithms (e.g. genetic algorithms, particle swarm optimization, and variants on gradient descent) in attempting to optimizing revenue using on-line adaptation of random discounting. 


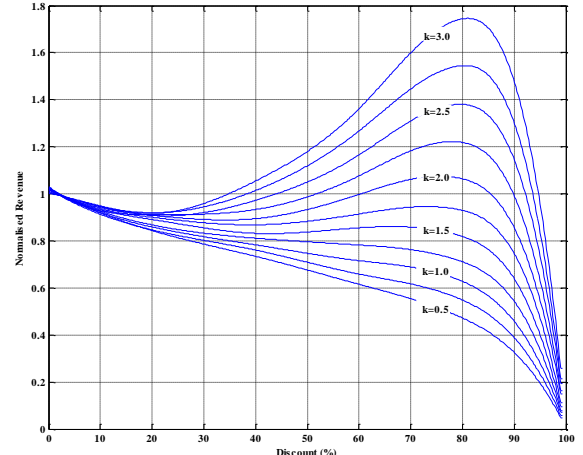

(a)

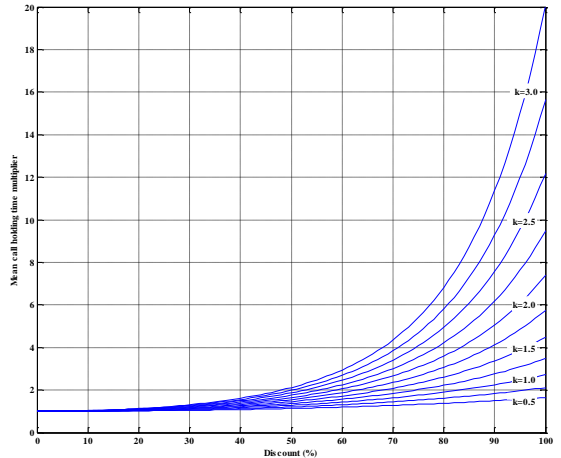

(b)

Fig. 24. (a) Normalized revenue versus discount curves for various call duration model parameter (k) values and (b) associated mean call duration versus discount curves

Fig. 24 (a) examines under what conditions our model would result in a scenario where optimization might be a desirable approach by considering the dependence on network wide revenue on the (k) parameter in the call duration model (3). Whilst a secondary maximum appears in the revenue curve for values of $\mathrm{k}$ greater than approximately $k=1.5$, these do not actually result in revenue gain for the operator until we consider $k$ values greater than approximately $k=1.8$. Using Fig. 24 (b) we can see that values of $k$ greater than 1.8, result in a call duration model where the mean call duration is likely to have very large value (i.e. greater than 5 to 6 times the mean call duration of the "no discount") when very large discount are offered to subscribers. It is hard to argue that this level of revenue elasticity is very likely to be realistic. The conclusion to be drawn based on the results of our model is that for a fixed population (of subscribers \agents) model there does not appear to be a scope for the practical use of optimization algorithms.

\section{CONCLUSION}

In this paper, we present an agent-based model for the simulation of the subscribers' calling behavior in a dynamically priced mobile network offer voice telephony services. The behavior of the agents in the model was developed through the analysis of CDRs generated from a real mobile network in Uganda in which dynamic pricing was deployed. We subsequently utilized the developed model to investigate a number of alternative dynamic pricing strategies using calling behavior models, a subscriber mobility model in Uganda and a social linkage \network model.

The most significant conclusion drawn from the results is that, short of offering no or very little discount to subscribers, the model suggests that all the forms of discounting considered will result in revenue loss to the network operator. This loss ranges from approximately $6 \%$ in the case of a subscriber centric discounting model where "high value" subscribers are offered enhanced random discounts to approximately $30 \%$ in the case of a basic load based discounting algorithm. In addition, the ABM provides a revenue-versus-mean discount approximation for the case where random discounting is deployed which allows estimation of likely revenue loss were such an algorithm deployed (operating at a specific mean discount value). We have also examined the potential for deploying optimization algorithms within the constraints of the model and the results indicate that these only potentially deliver a revenue growth scenario when the call duration model operates with 
Investigating the Revenue Generation Impact of Dynamic Pricing Algorithms for Mobile Voice Services $1: 33$

parameters which are likely to be unrealistic. The results obtained clearly suggest that operators can only minimize revenue loss, within the constraints of the model, by offering little or no discount. Clearly, in practice, this would make such a tariff virtually impossible to market and \or likely to result in significant subscriber dissatisfaction (and resultant outward subscriber churn with associated loss of revenue).

However, in practice, there are other effects or actions which we have not considered in out model which could offset these apparent revenue losses from the modelled fixed subscriber base. Firstly, it is likely that innovative and well marketed dynamic pricing tariff offerings will result in an increase in the subscriber base which commits to the dynamic pricing service through the process of inward subscriber "churn" [Ahn et al. 2006] (i.e. where subscribers from other networks change to the network offering dynamic pricing). Another option to address the potential revenue loss is to make the base (or "no discount" tariff within the dynamic priced service contract higher than the tariff for subscribers who remain outside the dynamic priced service. Through careful marketing, particular in countries where price sensitivity is a key driver and, perhaps, where there is a cultural attraction towards risk taking, subscribers will still be attracted into such a service in the hope that they will "gain" rather than "lose out" through the discounts which they encounter when making calls. From the network operator's perspective, the premium which might be added to the "no discount" tariff might simply be selected to offset the predicted revenue loss associated with the discounting algorithm, with revenue growth being achieved by the additional inward subscriber churn due to the dynamically priced service.

As noted in section 5.4, the development of optimization algorithms does not appear likely to deliver a commercially attractive offering within the constraints of a fixed subscriber based population. The deployment of such algorithms in practice is extremely challenging given the infrastructural and systems requirements to have access to and ability to process large volumes of near real time generated revenue information (either a cell, cluster, regional or national level). Additionally, such semior fully automated algorithms would introduce significant risks from an operator's perspective in terms of the potential for large revenue loss (or large subscriber dissatisfaction) should the algorithm malfunction in some manner. Lastly, from a purely algorithmic perspective, these revenue optimization algorithms would be attempting to carry out their revenue maximization in an extremely noisy feature space which might result in long convergence times (during which revenue generation will be sub-optimal) or the algorithm converging to a local revenue generation maximum rather than a global one.

In addition to further enhancing and testing our model with CDR data gathered from other dynamic pricing deployments, future work with this model will focus on a number of areas. Integration of a means of modelling subscriber "churn" (both inwards and outwards) into the ABM using realistic data on subscriber base changes during real DPS deployments would significantly enhance the realism of the ABM. In this work, we have utilized a data set gathered in a country where the subscriber base across all networks is almost completed prepaid and, in the case of the specific network whose CDRs were the basis for this work, virtually all prepaid subscribers opted into the dynamic pricing service. In other networks, particularly in industrially 
more developed regions, a far more significant portion of both the subscriber base but also more importantly the revenue generation base will be made up of postpaid subscribers (who tend to be far less price sensitive). In some cases significant proportions of such postpaid subscriber may not opt into a dynamic pricing service (if it is even offered to postpaid subscriber) but they will have an expectation that their grade of service will not be negatively impacted by the load increase associated with a DPS. Methodologies for modelling such subscribers and in particular constraints enforced on dynamic pricing algorithms as a result of a network operator's desire to maintain their grade of service also need to be investigated and integrated into the model. Finally, future work also needs to focus on using the developed ABM to predict the performance of semi- or fully automated revenue optimization algorithms in realistic scenarios where they can deliver revenue growth to operators while still offering subscribers attractive discount levels.

\section{ACKNOWLEDGMENTS}

This research is funded under the Enterprise Partnership Program of the Irish Research Council (IRC) with co-funding from Tango Telecom Limited. The authors would also like to thank the World Bank for providing them with access to data and images used in this research.

\section{REFERENCES}

Jae-Hyeon Ahn, Sang-Pil Han and Yung-Seop Lee 2006. Customer churn analysis: Churn determinants and mediation effects of partial defection in the Korean mobile telecommunications service industry. Telecommunications Policy 30, 552-568.

Réka Albert and Albert-László Barabási 2002. Statistical mechanics of complex networks. Reviews of Modern Physics 74, 47-97.

Hunt Allcott. 2009. Real Time Pricing and Electricity Markets. Harvard University, Cambridge, MA.

Hunt Allcott 2011. Rethinking real-time electricity pricing. Resource and Energy Economics 33, 820-842.

Robert Axelrod 1997. The Complexity of Cooperation: Agent-Based Models of Competition and Collaboration. Princeton University Press, Chichester, West Sussex.

Steven C. Bankes 2002. Agent-based modeling: A revolution? Proceedings of the National Academy of Sciences 99, 7199-7200.

Albert-László Barabási 2009. Scale-Free Networks: A Decade and Beyond. Science 325, 412-413.

Alain Barrat, Marc Barthélemy, Romualdo Pastor-Satorras and Alessandro Vespignani 2004. The architecture of complex weighted networks. National Academy of Sciences of the United States of America 101, 3747-3752.

Alain Barrat, Marc Barthélemy and Alessandro Vespignani 2005. The effects of spatial constraints on the evolution of weighted complex networks. Journal of Statistical Mechanics: Theory and Experiment, P05003.

Christopher L. Barrett, Keith R. Bisset, Stephen G. Eubank, Xizhou Feng and Madhav V. Marathe 2008. EpiSimdemics: An efficient algorithm for simulating the spread of infectious disease over large realistic social networks. In High Performance Computing, Networking, Storage and Analysis, 2008. SC 2008. International Conference for, 1-12.

Abd El-moniem Bayoumi, Mohamed Saleh, Amir F. Atiya and Heba Abdel Aziz 2013. Dynamic pricing for hotel revenue management using price multipliers. Journal of Revenue Pricing Management 12, 271285.

Richard A. Becker, Ramón Cáceres, Karrie Hanson, Ji Meng Loh, Simon Urbanek, Alexander Varshavsky and Chris Volinsky 2011. A Tale of One City: Using Cellular Network Data for Urban Planning. Pervasive Computing, IEEE 10, 18-26.

Richard Becker, Ramón Cáceres, Karrie Hanson, J. M. Loh, S. Urbanek, A. Varshavsky and C. Volinsky 2011. Clustering Anonymized Mobile Call Detail Records to Find Usage Groups. In Proceedings of the The First Workshop on Pervasive Urban Applications (PURBA)2011.

Federico Bergenti, Enrico Franchi and Agostino Poggi 2011. Agent-based Social Networks for Enterprise Collaboration. In Enabling Technologies: Infrastructure for Collaborative Enterprises (WETICE), 2011 20th IEEE International Workshops on, 25-28.

Vincent Blondel, Jean-Loup Guillaume, Renaud Lambiotte and Etienne Lefebvre 2008. Fast unfolding of communities in large networks. Journal of Statistical Mechanics: Theory and Experiment 2008, P10008.

Eric Bonabeau 2002. Agent-based modeling: Methods and techniques for simulating human systems. Proceedings of the National Academy of Sciences 99, 7280-7287.

Adrian W. Bowman and Adelchi Azzalini 1997. Applied Smoothing Techniques for Data Analysis : The Kernel Approach with S-Plus Illustrations: The Kernel Approach with S-Plus Illustrations. OUP Oxford. 
Investigating the Revenue Generation Impact of Dynamic Pricing Algorithms for Mobile Voice Services $1: 35$

Francesco Calabrese, Francisco Pereira, Giusy Di Lorenzo, Liang Liu and Carlo Ratti 2010. The Geography of Taste: Analyzing Cell-Phone Mobility and Social Events. In Proceedings of the 8th international conference on Pervasive Computing2010 Springer Berlin / Heidelberg, 22-37.

Julián Candia, Marta C González, Pu Wang, Timothy Schoenharl, Greg Madey and Albert-László Barabási 2007. Uncovering individual and collective human dynamics from mobile phone records.

Christian J. E. Castle and Andrew T. Crooks. 2006. Principles and Concepts of Agent-Based Modelling for Developing Geospatial Simulations. University College London, Torrington Place, London.

Manuel Cebrián, Alex Pentland and Scott Kirkpatrick 2010. Disentangling Social Networks inferred from Call Logs. CoRR abs / 1008.1357.

Kang Chaogui, Gao Song, Lin Xing, Xiao Yu, Yuan Yihong, Liu Yu and Ma Xiujun 2010. Analyzing and geo-visualizing individual human mobility patterns using mobile call records. In Geoinformatics, 2010 18th International Conference on, 1-7.

Emile J.L. Chappin and Maarten R. Afman 2013. An agent-based model of transitions in consumer lighting: Policy impacts from the E.U. phase-out of incandescents. Environmental Innovation and Societal Transitions 7, 16-36.

Steffen Christ 2011. Operationalizing Dynamic Pricing Models. Gabler, Wiesbaden GmbH, Wiesbaden.

Jaekwon Chung and Dong Li 2013. A simulation of the impacts of dynamic price management for perishable foods on retailer performance in the presence of need-driven purchasing consumers. $J$ Oper Res Soc.

Andrew Crooks, Christian Castle and Michael Batty 2008. Key challenges in agent-based modelling for geo-spatial simulation. Computers, Environment and Urban Systems 32, 417-430.

Koustuv Dasgupta, Rahul Singh, Balaji Viswanathan, Dipanjan Chakraborty, Sougata Mukherjea, Amit A Nanavati and Anupam Joshi 2008. Social ties and their relevance to churn in mobile telecom networks. In Proceedings of the 11th international conference on Extending database technology: Advances in database technology, Nantes, France2008 ACM, 1353424, 668-677.

Joan Morris Dimicco, Pattie Maes and Amy Greenwald 2003. Learning Curve: A Simulation-Based Approach to Dynamic Pricing 3, 245-276.

Alexandre Dolgui and Jean-Marie Proth 2010. Supply Chain Engineering: Useful Methods and Techniques. Springer.

Kevin P. Donovan and Aaron K. Martin 2014. The rise of African SIM registration: The emerging dynamics of regulatory change. First Monday; Volume 19, Number 2 - 3 February 2014.

E3. 2006. A Survey of Ttime-of-Use (TOU) Pricing and Demand-Response (DR) Programs. Energy \& Environmental Economics, San Francisco, CA.

Nathan Eagle, Yves-Alexandre de Montjoye and Luís M. A. Bettencourt 2009. Community Computing: Comparisons between Rural and Urban Societies Using Mobile Phone Data. In Computational Science and Engineering, 2009. CSE '09. International Conference on, 144-150.

Lars Ehrler, Martin Fleurke, Maryam Purvis, Bastin Tony and Roy Savarimuthu 2005. Agent-based workflow management systems (WfMSs), JBees: a distributed and adaptive WfMS with monitoring and controlling capabilities. Journal of Information Systems and E-Business Management 4, 18.

Abdulrahman M El-Sayed, Peter Scarborough, Lars Seemann and Sandro Galea 2012. Social network analysis and agent-based modeling in social epidemiology. Epidemiologic Perspectives \& Innovations 9.

Paul Expert, Tim Evans, Vincent Blondel and Renaud Lambiotte 2011. Uncovering space-independent communities in spatial networks. National Academy of Sciences 108, 7663-7668.

Pedro Faria and Zita Vale 2011. Demand response in electrical energy supply: An optimal real time pricing approach. Energy 36, 5374-5384.

Ahmad Faruqui 2010. The Ethics of Dynamic Pricing. The Electricity Journal 23, 13-27.

Ahmad Faruqui and Stephen George 2005. Quantifying Customer Response to Dynamic Pricing. The Electricity Journal 18, 53-63.

Peter C. Fishburn and Andrew M. Odlyzko 1998. Dynamic behavior of differential pricing and quality of service options for the Internet. In Proceedings of the Proceedings of the first international conference on Information and computation economies, Charleston, South Carolina, USA1998 ACM, 289024, 128139.

Elena Fitkov-Norris and Ahmad Khanifar 2000. Dynamic pricing in mobile communication systems. In $3 G$ Mobile Communication Technologies, 2000. First International Conference on (Conf. Publ. No. 471), 416-420.

Elena Fitkov-Norris and Ahmad Khanifar 2001. Dynamic pricing in cellular networks, a mobility model with a provider-oriented approach. In 3G Mobile Communication Technologies, 2001. Second International Conference on (Conf. Publ. No. 477), 63-67.

Santo Fortunato 2010. Community detection in graphs. Physics Reports 486, 75-174.

Enrique Frías-Martínez, Graham Williamson and Vanessa Frías-Martínez 2011. An Agent-Based Model of Epidemic Spread Using Human Mobility and Social Network Information. In Privacy, security, risk 
and trust (passat), 2011 ieee third international conference on and 2011 ieee third international conference on social computing (socialcom), 57-64.

Rob van Gerwen, Saskia Jaarsma, Rob Wilhite and KEMA. 2006. Smart Metering. Leonardo Energy, Brussels, Belgium.

Nigel Gilbert 2008. Agent-Based Models. SAGE Publications.

Marta C. Gonzalez, Cesar A. Hidalgo and Albert-Laszlo Barabasi 2008. Understanding individual human mobility patterns. Nature 453, 779-782.

Dan Guo, Bo Ren and Cheng Wang 2008. Integrated Agent-Based Modeling with GIS for Large Scale Emergency Simulation. In Advances in Computation and Intelligence, Lishan Kang, Zhihua Cai, Xuesong Yan and Yong Liu (Eds.). Springer Berlin Heidelberg, 618-625.

Lynne Hamill and Nigel Gilbert. 2010. A Simple but More Realistic Agent-based Model of a Social Network. Centre for Research in Social Simulation, University of Surrey, Guildford, Surrey.

Lynne Hamill and Nigel Gilbert. 2010. Simulating Large Social Networks In Agent-Based Models: A Social Circle Model. Centre for Research in Social Simulation, University of Surrey, UK.

Mohammed Bassam Hassouna. 2012. Agent Based Modelling and Simulation: An Examination of Customer Retention in the UK Mobile Market. Ph.D. Dissertation. Brunel University,

Yukio Hayashi 2006. A Review of Recent Studies of Geographical Scale-Free Networks. IPSJ Digital Courier 2, 155-164.

Cesar A. Hidalgo and C. Rodriguez-Sickert 2008. The dynamics of a mobile phone network. Physica A: Statistical Mechanics and its Applications 387, 3017-3024.

Theus Hossmann, Thrasyvoulos Spyropoulos and Franck Legendre 2011. A complex network analysis of human mobility. In Computer Communications Workshops (INFOCOM WKSHPS), 2011 IEEE Conference on, 876-881.

Alexander Ihler. 2007. Kernel Density Estimation Toolbox for MATLAB. Retrieved June 21, 2014 from http://www.ics.uci.edu/ ihler/code/kde.html

Sibren Isaacman, Richard Becker, Ram 'on C'aceres, Stephen Kobourov, Margaret Martonosi, James Rowland and Alexander Varshavsky 2011. Identifying important places in people's lives from cellular network data. In Proceedings of the 9th international conference on Pervasive computing, San Francisco, USA2011 Springer-Verlag, 2021988, 133-151.

Sibren Isaacman, Richard Becker, Ramón Cáceres, Margaret Martonosi, James Rowland, Alexander Varshavsky and Walter Willinger 2012. Human mobility modeling at metropolitan scales. In Proceedings of the Proceedings of the 10th international conference on Mobile systems, applications, and services, Low Wood Bay, Lake District, UK2012 ACM, 2307659, 239-252.

ITU. 2014. The World in 2014: ICT Facts and Figures. (March 2014). Retrieved June 21, 2014 from http://www.itu.int/en/ITU-D/Statistics/Pages/facts/default.aspx

Katrina Jessoe, David Rapson and Jeremy Blair Smith. 2012. The Effect of a Mandatory Time-of-Use Pricing Reform on Residential Electricity Use. Department of Economics, Boston University.

Woo-Sung Jung, Fengzhong Wang and H.Eugene Stanley 2008. Gravity model in the Korean highway. Europhysics Letters 81, 48005.

P. K. Kannan and Praveen K. Kopalle 2001. Dynamic Pricing on the Internet: Importance and Implications for Consumer Behavior. International Journal of Electronic Commerce 5, 63-83.

Rob Kitchin and Martin Dodge 2000. 'Placing' cyberspace : geography, community and identity 1, 25-46.

Benoît Leloup and Laurent Deveaux 2001. Dynamic Pricing on the Internet: Theory and Simulations 1, 265-276.

Yuri Levin, Jeff McGill and Mikhail Nediak 2007. Price Guarantees in Dynamic Pricing and Revenue Management. Operations Research 55, 75-97.

David Liben-Nowell, Jasmine Novak, Ravi Kumar, Prabhakar Raghavan and Andrew Tomkins 2005. Geographic routing in social networks. National Academy of Sciences 102, 11623-11628.

Gregory Madey, Yongqin Gao, Vincent Freeh, Renee Tynan and Chris Hoffman 2003. Agent-Based Modeling and Simulation of Collaborative Social Networks. In Ninth Americas Conference on Information Systems, Tampa, FL, USA.

Federico Liévano Martínez and Yris Olaya Morales. 2012. Agent-Based Simulation Approach to Urban Dynamic Modeling. Universidad Nacional de Colombia, Colombia.

Robin B. Matthews, Nigel G. Gilbert, Alan Roach, J. Gary Polhill and Nick M. Gotts 2007. Agent-based land-use models: a review of applications. Landscape Ecology 22, 1447-1459.

R. Preston McAfee and Vera te Velde. 2006. Dynamic Pricing in the Airline Industry. California Institute of Technology, Pasadena, CA.

Seyed Mohammadreza Mirsarraf and Ali Reza Mansoori 2008. Comparison of telecommunication service providers' pricing strategies for packet based services. In Telecommunications, 2008. IST 2008. International Symposium on, 30-35.

Andres Molina-Markham, George Danezis, Kevin Fu, Prashant Shenoy and David Irwin 2012. Designing Privacy-Preserving Smart Meters with Low-Cost Microcontrollers. In Financial Cryptography and Data Security, AngelosD Keromytis (Eds.). Springer Berlin Heidelberg, 239-253. 
Investigating the Revenue Generation Impact of Dynamic Pricing Algorithms for Mobile Voice Services $1: 37$

Amit A. Nanavati, Rahul Singh, Dipanjan Chakraborty, Koustuv Dasgupta, Sougata Mukherjea, Gautam Das, Siva Gurumurthy and Anupam Joshi 2008. Analyzing the Structure and Evolution of Massive Telecom Graphs. Knowledge and Data Engineering, IEEE Transactions on 20, 703-718.

Javad Nasiry and Ioana Popescu 2011. Dynamic Pricing with Loss-Averse Consumers and Peak-End Anchoring. Operations Research 59, 1361-1368.

M. E. J. Newman 2006. Modularity and community structure in networks. Proceedings of the National Academy of Sciences 103, 8577-8582.

Cynthia Nikolai and Gregory Madey 2009. Tools of the Trade: A Survey of Various Agent Based Modeling Platforms. Journal of Artificial Societies and Social Simulation 12.

Michael J. North, Charles M. Macal, James St. Aubin, Prakash Thimmapuram, Mark Bragen, June Hahn, James Karr, Nancy Brigham, Mark E. Lacy and Delaine Hampton 2010. Multiscale agent-based consumer market modeling. Complex. 15, 37-47.

Anastasios Noulas, Salvatore Scellato, Renaud Lambiotte, Massimiliano Pontil and Cecilia Mascolo 2012. A Tale of Many Cities: Universal Patterns in Human Urban Mobility. PLoS ONE 7, e37027.

Alan Olivré. 2004. Call Admission Control and Dynamic Pricing in a GSM/GPRS Cellular Network. Master's thesis. University of Dublin, Trinity College, Dublin, Ireland.

Jukka-Pekka Onnela, Jari Saramäki, Jorkki Hyvonen, Gyorgy Szabó, David Lazer, Kimmo Kaski, János Kertész and Albert-László Barabási 2007. Structure and tie strengths in mobile communication networks. Proceedings of the National Academy of Sciences 104, 7332-7336.

Dawn C. Parker, Thomas Berger and Steven M. Manson 2001. Agent-Based Models of Land-Use and Land-Cover Change. In Proceedings of the Report and Review of an International Workshop, Irvine, California, USA2001, William J. McConnell (Eds.).

Dawn C. Parker, Steven M. Manson, Marco A. Janssen, Matthew J. Hoffmann and Peter Deadman. 2002. Multi-Agent Systems for the Simulation of Land-Use and Land-Cover Change: A Review. Forthcoming, Annals of the Association of American Geographers, Irvine, California, USA.

Achille Pattavina and Alessandra Parini 2005. Modelling voice call interarrival and holding time distributions in mobile networks. In Proceedings of the 19th International Teletraffic Congress2005 Beijing University of Posts and Telecommunications Press.

Utpal Paul, Anand Prabhu Subramanian, Milind Madhav Buddhikot and Samir R. Das 2011. Understanding traffic dynamics in cellular data networks. In INFOCOM, 2011 Proceedings IEEE, 882 890.

Ioana Popescu and Yaozhong Wu 2007. Dynamic Pricing Strategies with Reference Effects. Operations Research 55, 413-429.

Ye Qi, Zhu Tian, Hu Deyong, Wu Bin, Du Nan and Wang Bai 2008. Cell phone mini challenge award: Social network accuracy exploring temporal communication in mobile call graphs. In Visual Analytics Science and Technology, 2008. VAST '08. IEEE Symposium on.

Zhang Qin, Wang Xifan and Fu Min 2009. Optimal implementation strategies for critical peak pricing. In Energy Market, 2009. EEM 2009. 6th International Conference on the European, 1-6.

William Rand and Roland T. Rust 2011. Agent-based modeling in marketing: Guidelines for rigor. International Journal of Research in Marketing 28, 181-193.

Lamjed Ben Said, Thierry Bouron and Alexis Drogoul 2002. Agent-based interaction analysis of consumer behavior. In Proceedings of the Proceedings of the first international joint conference on Autonomous agents and multiagent systems: part 1, Bologna, Italy2002 ACM, 544787, 184-190.

Pedram Samadi, Amir-Hamed Mohsenian-Rad, Robert Schober, Vincent W.S. Wong and Juri Jatskevich 2010. Optimal Real-Time Pricing Algorithm Based on Utility Maximization for Smart Grid. In Smart Grid Communications (SmartGridComm), 2010 First IEEE International Conference on, 415-420.

Eleni Samanidou, Elmar Zschischang, Dietrich Stauffer and Thomas Lux 2007. Agent-based models of financial markets. Reports on Progress in Physics 70.

SAS. 2010. How can we prevent the churn of our most profitable customers? . Retrieved June 21, 2014 from http://www.sas.com/resources/solution-brief/104696_0810.pdf

Nina Schwarz and Andreas Ernst 2009. Agent-based modeling of the diffusion of environmental innovations - An empirical approach. Technological Forecasting and Social Change 76, 497-511.

Mukund Seshadri, Sridhar Machiraju, Ashwin Sridharan, Jean Bolot, Christos Faloutsos and Jure Leskove 2008. Mobile call graphs: beyond power-law and lognormal distributions. In Proceedings of the Proceedings of the 14th ACM SIGKDD international conference on Knowledge discovery and data mining, Las Vegas, Nevada, USA2008 ACM, 1401963, 596-604.

H. M. Singer, I. Singer and H. J. Herrmann 2009. Agent-based model for friendship in social networks. Physical Review E 80, 026113.

Chaoming Song, Zehui Qu, Nicholas Blumm and Albert-László Barabási 2010. Limits of Predictability in Human Mobility. Science 327, 1018-1021. 
Victor Soto and Enrique Frías-Martínez 2011. Robust Land Use Characterization of Urban Landscapes using Cell Phone Data. In Proceedings of the The First Workshop on Pervasive Urban Applications (PURBA)2011.

William Tepfenhart, Wang Jiacun and Daniela Rosca 2009. Agent based emergency response workflow management. In Service Operations, Logistics and Informatics, 2009. SOLI '09. IEEE/INFORMS International Conference on, 140-146.

Vikrant Tomar, Himanshu Asnani, Abhay Karandikar, Vinay Chander, Swati Agrawal and Prateek Kapadia 2010. Social network analysis of the Short Message Service. In Communications (NCC), 2010 National Conference on, 1-5.

Paul Twomey and Richard Cadman 2002. Agent-based modelling of customer behaviour in the telecoms and media markets. info $4,8$.

UCC. 2012. 2011/12 Half Year Market Performance Review. (November 2012). Retrieved June 21, 2014 from http://www.ucc.co.ug/files/downloads/2011$12 \% 20$ Half\%20Year\%20Market\%20Performance\%20Review\%20New\%20(1).pdf

Marcos R. Vieira, Vanessa Frías-Martínez, Nuria Oliver and Enrique Frías-Martínez 2010. Characterizing Dense Urban Areas from Mobile Phone-Call Data: Discovery and Social Dynamics. In Social Computing (SocialCom), 2010 IEEE Second International Conference on, 241-248.

Fergal Walsh and Alexei Pozdnoukhov 2011. Spatial structure and dynamics of urban communities. In Proceedings of the The First Workshop on Pervasive Urban Applications (PURBA)2011.

Han Wang and Liam Kilmartin 2014. Comparing Rural and Urban Social and Economic Behavior in Uganda: Insights from Mobile Voice Service Usage. Journal of Urban Technology 21, 61-89.

Pu Wang, Marta C. González, César A. Hidalgo and Albert-László Barabási 2009. Understanding the Spreading Patterns of Mobile Phone Viruses. Science 324, 1071-1076.

Daniel Willkomm, Sridhar Machiraju, Jean Bolot and Adam Wolisz 2008. Primary Users in Cellular Networks: A Large-Scale Measurement Study. In New Frontiers in Dynamic Spectrum Access Networks, 2008. DySPAN 2008. 3rd IEEE Symposium on, 1-11.

Saravut Yaipairoj and Fotios C. Harmantzis 2004. A dynamic pricing model for data services in GPRS networks. In Global Telecommunications Conference Workshops, 2004. GlobeCom Workshops 2004. IEEE, 453-458.

Qi Ye, Bin Wu, Lijun Suo, Tian Zhu, Chao Han and Bai Wang 2009. TeleComVis: Exploring Temporal Communities in Telecom Networks. In Machine Learning and Knowledge Discovery in Databases, Wray Buntine, Marko Grobelnik, Dunja Mladenić and John Shawe-Taylor (Eds.). Springer Berlin Heidelberg, 755-758.

Yihong Yuan, Martin Raubal and Yu Liu 2011. Correlating mobile phone usage and travel behavior - A case study of \{Harbin, China\}. Computers, Environment and Urban Systems.

Hui Zang and Jean C. Bolot 2007. Mining call and mobility data to improve paging efficiency in cellular networks. In Proceedings of the 13th annual ACM international conference on Mobile computing and networking, Montréal, Québec, Canada2007 ACM, 1287868, 123-134.

Tao Zhang and David Zhang 2007. Agent-based simulation of consumer purchase decision-making and the decoy effect. Journal of Business Research 60, 912-922. 